\title{
Incorporating uncertainty of management costs in sensitivity analysis of matrix population models
}

YACOV SALOMON,${ }^{*} \dagger$ MICHAEL A. McCARTHY,$\dagger$ PETER TAYLOR, ${ }^{*}$ AND BRENDAN A. WINTLE $\dagger$

* Department of Mathematics and Statistics, University of Melbourne, Parkville VIC 3010, Australia.

$\dagger$ School of Botany, University of Melbourne, Parkville VIC 3010, Australia.

Running title: Accounting for cost uncertainty

Keywords: conservation, uncertainty, stochastic optimization, portfolio theory, population management, perturbation analysis, decision theory.

Word count: 5360

Correspondence: Yacov Salomon

Department of Mathematics and Statistics

University of Melbourne

Parkville VIC 3010, Australia

Email: ysalomon@pgrad.unimelb.edu.au 


\section{Abstract}

The importance of accounting for economic costs when making environmental management decisions subject to resource constraints has been increasingly recognized in recent years. In contrast, uncertainty associated with such costs has often been ignored. Here, we introduce and develop a method, motivated by economic theory, that accounts for the uncertainty in population management decisions. We consider the case where, rather than taking fixed values, model parameters are random variables to represent the situation when the parameters are not precisely known. Hence, the outcome is not precisely known

either. Instead of maximising the expected outcome we choose to maximize the probability of obtaining an outcome above a threshold of acceptability. We derive explicit analytical expressions for the optimal allocation and its associated probability, as a function of the threshold of acceptability, where the model parameters are distributed according to the normal and the uniform distributions. To illustrate our approach we revisit a previous study that incorporated cost-efficiency analysis in management decisions based on perturbation analysis of matrix population models. Incorporating their derivation into our framework, we extend their model to address potential uncertainties. We then apply these results to two case studies; management of a Koala population (Phascolarctos cinereus), and conservation of an olive-ridley sea turtle population (Lepidochelys olivacea). From our results, for low aspirations, the optimal strategy is obtained by diversifying the allocation. Conversely, for high aspirations, the budget is directed towards the management action with the highest potential effect on the population. Furthermore, the exact optimal allocation was sensitive to the choice of uncertainty model. These results highlight the importance of accounting for uncertainty when making decisions, and suggest that more effort should be placed on understanding the distributional characteristics of 
such uncertainty. Our approach provides a tool to inform better decision-making.

\section{Introduction}

One of the key problems occupying the conservation community is how to allocate limited resources between multiple possible management actions. This problem applies to a range of conservation questions including the design of reserve networks (Possingham et al. 2001; Moore et al. 2004; McCarthy et al. 2005), conservation of threatened species (McCarthy et al. 2008; Joseph et al. 2009), and managing threatened, migratory or invasive species (Baxter et al. 2006; Martin et al. 2007).

Several approaches to cost-efficiency analysis have been proposed, depending on the particular circumstances (e.g., Wilson et al. 2006; Baxter et al. 2006; Bode et al. 2008). However, these approaches typically use only point estimates of expected costs and benefits, ignoring associated uncertainties. Factors contributing to uncertainty include the lack of information, as well as inherent variability. Indeed the magnitude of such uncertainties may be large, or vary considerably between competing conservation actions (e.g., Moore et al. 2004). Ignoring uncertainties exposes one to overconfidence and increased likelihood of failure (Burgman 2005).

While rarely considered in the conservation literature, cost uncertainty plays a major role in economic decision theory, where it is often termed risk. Dating back more than half a century, Roy (1952) defined the safety first principle to tackle the problem of making economic decisions when uncertainty is present. The safety first principle argues that, when faced with uncertainty, one should choose the action that maximizes the probability 
of a satisfactory outcome. This principle is reflected in prevalent use of Value at Risk (VaR) to measure financial risk (McNeil et al. 2005).

Another important contribution to the problem of making economic decisions under conditions of uncertainty was the seminal work by (Markowitz 1952 1991) on portfolio selection. Portfolio theory is concerned with the problem of allocating a financial resource (a budget) between various risky investment options termed assets (stocks, bonds etc.,). The expected return of an asset is defined as the mean change in its value over a given time step. The risk of an asset is defined as the uncertainty associated with its return, commonly measured by its standard deviation.

A portfolio is a linear combination of a set of assets, where the weights associated with each asset represent the fraction of the total resource invested (allocated) in each asset. The portfolio problem is then to determine the value of those weights. Reflecting the notion that individuals prefer higher returns and lower risk, portfolio theory states that the optimal allocation is that which maximizes the expected returns of a portfolio given a set level of risk, or equivalently, minimizes the risk given a set expected return. The optimal allocation reflects a trade-off between higher expected returns and lower risk. An important result of portfolio theory is that diversifying allocations, that is distributing the budget among multiple investment options, offers a way to mitigate risk. The extent of diversification reflects the expected and standard deviation of returns, the degree of correlation between the different assets and the risk appetite of the investor.

Portfolio theory has been applied in the context of environmental management a limited number of times (e.g., Springer 2003; Edwards et al. 2004; McCarthy et al. 2010). In this context, assets are replaced by different management actions. Just as in the classical 
portfolio theory, in most environmental management applications, there are uncertainties associated with outcomes, and these have previously been assumed to follow a normal distribution (but see McCarthy et al. 2010). This assumption is reasonable when the underlying random variables are indeed normally distributed. The normal assumption is also a good approximation when, by the central limit theorem, the portfolio consists of a large enough number of actions that are independent and identically distributed. In some cases, however, such as when considering only a small number of competing actions, other distributions are more appropriate. In this paper, motivated by Roys safety first principle, we re-formulate the portfolio problem in a more general form where the optimal allocation is defined as the allocation that maximizes the probability of obtaining a return above a threshold of acceptability. Determining the optimal allocation in the sense is indeed equivalent to the classical portfolio problem when assets are independent, and the uncertainty is assumed to follow a normal distribution (Roy 1952, and Lemma 1.2 in Supporting Information).

We apply the notion of maximising the probability of a favourable outcome, and the portfolio approach, to the problem of conservation of threatened species. Such problems are typically analysed using matrix population models and perturbation analysis (Caswell 2001). The population matrix model is given by the set of difference equations $\mathbf{n}(t+1)=$ $\mathbf{A n}(t)$, where $\mathbf{n}(t)$ is a vector denoting the number of individuals in the population, classified according to age, life stage, or some other characteristics. The elements of the matrix $\mathbf{A}$ denote transition rates between the different classes. The long term (asymptotic) growth rate of the population is given by the dominant eigenvalue of $\mathbf{A}$, denoted here by $\lambda$. A population is said to be asymptotically increasing if the asymptotic growth rate is greater than $1(\lambda>1)$, and decreasing otherwise. 
Perturbation analysis consists of examining the change (sensitivity) or proportional change (elasticity) of the asymptotic growth rate with respect to changes in the elements of $\mathbf{A}$, induced by each conservation action. Such analysis, however, only accounts for the biological aspects of the conservation problem.

In order to account for the economical aspects, Baxter et al. (2006) combined perturbation analysis with a cost-efficiency analysis. According to such a formulation, the optimal action would be the one offering the greatest change in the asymptotic growth rate of the population per dollar spent. However, the formulation by Baxter et al. (2006) does not account for uncertainty. Applying our suggested approach the optimal allocation is then the one for which a minimally-acceptable change in the asymptotic growth rate of the population, per dollar spent, occurs with the greatest probability. We illustrate our approach numerically by applying it to the conservation of two endangered species; a Koala population (Phascolarctos cinereus) on Snake Island, and an olive-ridley sea turtle population (Lepidochelys olivacea) in Orissa, India.

\section{Methods}

\section{Safety first portfolio formulation}

Many environmental managers are faced with the task of deciding how to allocate limited resources between $n$ possible independent management actions in order to achieve some desirable outcome. For simplicity we can think of the limited resources in terms of a total budget. . Let the random variable $R_{i}$ denote the change in the overall management 
outcome per dollar spent on action $i$.. Then, assuming that the $R_{i}$ are additive and independent of the amount allocated, the outcome of a management plan, a mixture of $n$ management actions, is

$$
R(\mathbf{x})=\sum_{i=1}^{n} x_{i} R_{i}
$$

where $x_{i}$ is the fraction of the total budget allocated to action $i\left(\sum_{i=1}^{n} x_{i}=1\right)$. Equation (1) describes the outcome of the portfolio of $n$ management actions defined by the vector $\mathbf{x}=\left(x_{1}, \ldots, x_{n}\right)$. The implications of the additive and independence assumptions will be further discussed in the Results section.

Suppose one defines a minimal threshold of acceptability, $\rho$. The optimal allocation is then the one that maximizes the probability of obtaining a portfolio outcome above $\rho$. Mathematically, the optimal allocation is given by the optimization problem

$$
\arg \max _{\mathbf{x}} P\{R(\mathbf{x}) \geq \rho\}
$$

subject to $0<x_{i}<1 i=1, \ldots, n$, and $\sum_{i=1}^{n} x_{i}=1$. By varying the value of $\rho$ over its entire range, one is able to explore the trade-off between insisting on a higher threshold of acceptability and the probability of achieving it.

Obtaining the optimal allocation in Eq. (2) requires, in general, first obtaining an expression for the probability distribution of $R(\mathbf{x})$. In the case when the weighted marginal efficiencies , $\left\{x_{i} R_{i}\right\}$, are independent, the distribution of $R(\mathbf{x})$ is given by the convolution of their respective distributions.

In this paper we illustrate the application of the above formulation in the context of management of threatened species, based on perturbation analysis of matrix population 
models. In both case studies one is faced with the decision of allocating resources between two independent management actions $(n=2)$. To illustrate our formulation, for each case study, we consider two different models of uncertainty, with the random marginal efficiencies $R_{i}$ either normally or uniformly distributed..

We solve the non-linear constrained optimization problem Eq. (2) using the KarushKuhn-Tucker (KKT) conditions (Bertsekas 1999) to obtain analytical expressions for the optimal allocation, and the associated probability of satisfying the threshold of acceptability, for both uncertainty models (see Supporting Information for a detailed analysis).

\section{Application to perturbation analysis}

We are given some, real, nonnegative, age- or stage-classified, population projection matrix A. Such matrices are, in general, irreducible and primitive (Caswell 2001). Thus, by Perron-Frobenius theory (Caswell 2001), the projection matrix must have a real and positive dominant eigenvalue, the expected long term population growth rate, which we denote by $\lambda$. Furthermore, the dominant eigenvalue is simple and the right and left eigenvectors ( $\mathbf{w}$ and $\mathbf{v}$ respectively) are also real, strictly positive and unique up to multiplication by a constant.

Let $a_{j k}$ be the elements of $\mathbf{A}$, and assume that some "lower level" parameters $\ell_{i}(i=$ $1, \ldots, n)$ affect one or more elements $a_{j k}$. The sensitivity of $\lambda$ to changes in $\ell_{i}$ is defined as

$$
\frac{\partial \lambda}{\partial \ell_{i}}=\sum_{j, k} \frac{\partial \lambda}{\partial a_{j k}} \frac{\partial a_{j k}}{\partial \ell_{i}}=\sum_{j, k} \frac{v_{j} w_{k}}{\mathbf{v} \cdot \mathbf{w}} \frac{\partial a_{j k}}{\partial \ell_{i}}
$$

where $\mathbf{w}$ and $\mathbf{v}$ are the right and left eigenvectors of $\lambda$ (Caswell 2001). 
In the context of environmental management, the lower level parameter $\left(\ell_{i}\right)$ denotes the effect of a particular management action on different characteristics of the population dynamics. For example, two such lower level parameters of particular interest are the survival $(s)$ associated with the diagonal and off diagonal elements of $\mathbf{A}$, and fecundity $(f)$ associated with the first row elements. Substituting $s$ or $f$ for $\ell_{i}$, in Eq. (3), we obtain the sensitivity of $\lambda$ to changes in the survival and fecundity respectively. The environmental decision is then a question of how much to invest in managing fecundity versus managing survival.

In order to incorporate costs in sensitivity analysis Baxter et al. (2006) assumed that, denoting the cost associated with management action $i$ by $C_{\ell_{i}}$, the marginal cost, $\partial C_{\ell_{i}} / \partial \ell_{i}$, is independent of other costs. Such an approximation is valid when the cost function is everywhere differentiable and approximately linear in a small neighborhood of any value of the parameter. They then defined a deterministic marginal efficiency as the change in the long-term population growth rate for unit management investment in life-history parameter $\ell_{i}$

$$
r_{\ell_{i}}=\frac{\partial \lambda}{\partial C_{\ell_{i}}}=\frac{\partial \lambda / \partial \ell_{i}}{\partial C_{\ell_{i}} / \partial \ell_{i}},
$$

Using Eq. (4) to obtain marginal efficiencies for each of a set of competing management actions, Baxter et al. (2006) suggests that the decision rule should be to choose the management strategy with the highest marginal efficiency. This is equivalent to choosing the strategy that offers the greatest expected effect on the population per dollar spent. Note, however, that the analysis thus far has only considered point estimates; the expression for the marginal efficiencies of each strategy, Eq. (4), ignores uncertainties.

To incorporate uncertainty we embed the model suggested by Baxter et al. (2006) into 
theportfolio formulation outlined above. In particular, we set the costs, and thus the marginal efficiencies, to be random variables in Eq. (4). We then construct a portfolio of such random marginal efficiencies, as in Eq. (1), and use the analytical expressions derived to obtain the optimal allocation as well as the probability of satisfying the associated minimally-acceptable marginal efficiency.

\section{Case study: The koala population on Snake Island}

While declining in parts of its range, some koala populations (Phascolarctos cinereus) are in fact over-abundant. The population on Snake Island (Victoria, Australia) is a case of the latter. Therefore the management objective is to reduce the population size, or equivalently decrease the long-term growth rate $\lambda$ of the population. McLean (2003) constructed a $9 \times 9$ stage-classified population projection matrix for the koala population on Snake Island (Table 1). Two alternative management actions were considered (Baxter et al. 2006). The first, translocation of individuals to the mainland, affected the underlying survival rate $s$. The second, sub-dermal contraception (Middleton et al. 2003), affected the underlying fecundity rate $f$. Both these strategies were assumed to target individuals with equal probability across stages $2-9$. We then parameterize the matrix in Table 1 (as per Baxter et al. 2006) as follows 


$$
\left(\begin{array}{cccccc}
0 & w_{2} f & w_{3} f & \cdots & w_{8} f & w_{9} f \\
\gamma_{1} q_{1} s & \left(1-\gamma_{2}\right) q_{2} s & 0 & \cdots & 0 & 0 \\
0 & \gamma_{2} q_{2} s & \left(1-\gamma_{3}\right) q_{3} s & \ddots & 0 & 0 \\
\vdots & \ddots & \ddots & \ddots & \vdots & \vdots \\
0 & 0 & 0 & \ddots & \left(1-\gamma_{8}\right) q_{8} s & 0 \\
0 & 0 & 0 & \ldots & \gamma_{8} q_{8} s & \left(1-\gamma_{9}\right) q_{9} s
\end{array}\right)
$$

where, $w_{i}$ and $q_{i}$ relate the stage-specific elements in the matrix in Table 1 with respect to the underlying fecundity rate $(f)$, and survivorship rate $(s)$, respectively. The parameter $\gamma_{i}$ is the proportion of surviving individuals in stage $i$ that advance to the next stage $\left(\gamma_{1}=1\right.$, and $\left.\gamma_{9}=0\right)$. As a point of reference, we set $w_{9}=q_{9}=1$ and so $f=0.0118$ and $s=0.6090$.

The dominant eigenvalue of the population matrix can be calculated to be $\lambda=1.04074$, and from Eq. (3) the sensitivities to change in fecundity and survival are $\partial \lambda / \partial f=21.0844$, and $\partial \lambda / \partial s=1.3010$ respectively. The expressions for the marginal efficiencies, Eq. (4), are hence given by (see Baxter et al. 2006, and Supporting Information for detailed derivation)

$$
\begin{aligned}
R_{f} & =\frac{\partial \lambda}{\partial f} \frac{f}{c_{f} N_{2-9}}=\frac{0.2488}{c_{f} N_{2-9}} \\
R_{s} & =\frac{\partial \lambda}{\partial s} \frac{s}{c_{s} N_{2-9}}=\frac{0.7923}{c_{s} N_{2-9}}
\end{aligned}
$$

where $c_{f}$ and $c_{s}$ are the management costs per (female) koala for each strategy, and $N_{2-9}$ is the number of individuals in stages 2-9.

Harbutt (2004) estimated the management costs per (female) koala to be between $\$ 100$ and $\$ 120$ for contraception $c_{f}$, and between $\$ 300$ and $\$ 400$ for translocation $c_{s}$. 
Baxter et al. (2006) chose to use the mid point of the reported ranges as the point estimates for obtaining uncertainty-free marginal efficiency values in eqns. (6) and (7). In contrast, as outlined earlier, our approach incorporates the reported uncertainty by considering $c_{f}$ and $c_{s}$, and therefore $R_{f}$ and $R_{s}$, to be independent random variables. Given the lack of information regarding the nature of the distributions that characterizes the uncertainties associated with this problem, we choose to fit the distributions over the marginal efficiencies rather than the costs since it offers greater mathematical tractability.

In particular, we examine two general types of uncertainty. In the first we assumed that the marginal efficiency of each action follows a normal distribution, where the mean is given by the marginal efficiency corresponding to the mid point of the respective interval of reported cost, and the variance is given by assuming the intervals for the costs represent probability intervals of varying value of percentiles $(20 \%, 50 \%, 90 \%, 95 \%$ or $99 \%)$. In the second case, we assumed that the marginal efficiencies are distributed uniformly over the interval corresponding to the respective cost interval.

Next we constructed the portfolio. For the special case of two competing management actions, Eq. (1) is reduced to

$$
R(x)=x R_{f}+(1-x) R_{s}
$$

where $x$ is the fraction of total budget allocated to managing fecundity (sub-dermal contraception). We then use our derived analytical expression to obtain the optimal allocation under each of the two uncertainty models, for a given threshold marginal efficiency level $\rho$, and the associated probability. Repeating this process for a range of $\rho$ values provides the entire spectrum of interplay between the optimal allocation and 
the probability of satisfying the critical value.

\section{Case study: An olive ridley sea turtle population}

Most sea turtle nesting populations are depleted worldwide. While some populations are increasing as a result of successful conservation efforts, many nesting populations remain very low with little or no signs of recovery (Seminoff \& Shanker 2008). Ongoing threats to sea turtle populations include intentional and accidental harvest and the destruction of nesting habitat.

Two of the main sea turtle conservation management actions practiced worldwide are the inclusion on trawling vessels of Turtle Excluding Devices (TEDs), and protection of nesting habitat (Crouse et al. 1987; Crowder et al. 1994). Fisheries that have included TEDs have reported significant reduction in by-catch of adult sea turtles (Crouse et al. 1992). At the same time, protecting nesting beaches increases hatchlings' survival by reducing egg harvest, nest destruction, and hatchling disorientation from artificial lights (Shanker 1994). Using our safety first portfolio formulation we illustrate how one may go about allocating a limited budget between the two potential conservation actions.

To apply our proposed method one requires both a population matrix and estimates of conservation costs. Unfortunately, as far as we are aware, no sea turtle population exists for which such data has been published. Instead, we use conservation cost estimates for a population of olive ridley sea turtles (Lepidochelys olivacea) in Orissa, India (K. Shanker, personal communication), combined with published stage-based population model for loggerhead sea turtles (Caretta caretta) (Crowder et al. 1994). While borrowing parameters 
from a related species is done here for illustrative purposes only, this is in fact a common practice when data are unavailable.

Denoting by $s$ the underlying adult survival rate affected by inclusion of TEDs, and by $h$ the hatchling survival rate affected by protection of nesting habitat, we parameterize the stage population matrix (Table 2) as follows

$$
\left(\begin{array}{ccccc}
0 & f_{2} & f_{3} & f_{4} & f_{5} \\
h & \left(1-\gamma_{2}\right) q_{2} & 0 & 0 & 0 \\
0 & \gamma_{2} q_{2} & \left(1-\gamma_{3}\right) q_{3} s & 0 & 0 \\
0 & 0 & \gamma_{3} q_{3} s & \left(1-\gamma_{4}\right) q_{4} s & 0 \\
0 & 0 & 0 & \gamma_{4} q_{4} s & q_{5} s
\end{array}\right) .
$$

The parameter $q_{i}$ relates the stage-specific elements in the matrix (Table 2), to the underlying survivorship $s$, and $\gamma_{i}$ is the proportion of surviving individuals in stage $i$ that advance to the next stage $\left(\gamma_{1}=1\right.$, and $\left.\gamma_{5}=0\right)$. For convenience, we set $q_{5}=1$ and so $h=0.675$ and $s=0.8091$.

The dominant eigenvalue of the population matrix is $\lambda=0.9516$, and from Eq. (3) the sensitivities are $\partial \lambda / \partial h=0.0816$, and $\partial \lambda / \partial s=0.7719$. The estimated range of marginal costs for each conservation action, $\partial C_{h} / \partial h$ and $\partial C_{s} / \partial s$, are, between $\$ 3000$ and $\$ 150000$ for habitat protection, and between $\$ 48500$ and $\$ 8240000$ for inclusion of TEDs (see Supporting Information). Using these estimates in Eq. (4) we obtain expressions for the stochastic marginal efficiencies of each conservation action (detailed derivation in Supporting Information).

As for our koala example, we tested two uncertainty models, normal and uniform, for 
the parameters of interest. Constructing a portfolio, we solve for the optimal allocation and the corresponding probability of satisfying a particular threshold portfolio marginal efficiency (Figs. 3-4).

\section{Results}

The optimal allocation is independent of the choice of variance when uncertainties are represented by normal distributions (Figs. 1 and 3), provided that the ratio of the standard deviations of the two strategies remains constant (see Supporting Information for analytical justification). In contrast, the probability of satisfying a given critical threshold is, indeed, sensitive to the choice of variance (Figs. 1 and 3). Furthermore, decreasing the standard deviation in this way suggests a limit process whereby allocations for threshold marginal efficiency levels below the lower of the two expected marginal efficiencies are satisfied with probability approaching unity.

Conversely, allocations for threshold values above the higher of the two expected marginal efficiencies are satisfied with probability approaching zero as the standard deviation decreases. This somewhat intuitive result states that as the expected marginal efficiency of each strategy becomes more certain, we would be increasingly certain of obtaining marginal efficiency at the expected value.

Next we note that for positive values of $\rho$, that are below the expected marginal efficiency of all strategies, the optimal allocation is a diverse one. This is in agreement with the notion that spreading an investment over several uncertain assets mitigates risk, and traces back to the original motivation for the portfolio mean-variance theory (Markowitz 
1952).

Increasing the value of $\rho$ beyond the expected marginal efficiency of either action, the optimal allocation transitions to allocating the entire budget to a single strategy (see Supporting Information for analytical justification). Although initially this might seem counterintuitive, such a decision could be understood through the analogy of the roulette chance game. For a relatively low requirement for returns, risk could be mitigated in a roulette game by betting on multiple (often negatively correlated) events. As our requirements of return increase above the expected outcome (in a single roulette game the expected outcome is a small proportional loss) our approach shifts towards increasingly placing all our bets on the high risk and high return events (for example, placing all bets on a particular number). Such a strategy maximizes the probability of achieving a big gain in a single game, even though the probability of realizing such an event is small.

When the variance of the action with the least expected marginal efficiency is sufficiently large in comparison to the variance of the competing action, the entire budget allocation can switch between actions (Supporting Information equations S1.23 and S1.24). Indeed, such a transition occurrs for the koala population management example. At a threshold level of around $4.25 \times 10^{-6}$, the optimal allocation transitions from allocating the entire budget to managing survival, to allocating the entire budget to managing fecundity (Fig. 1). Note that in this case, the probability of satisfying the objective is very small, across all the variance assumptions, and therefore, these allocations would most likely be ineffective. However, in general, such transitions could occur for optimal allocations where the associated probabilities are meaningful.

When the ratio of the standard deviations of the two strategies changes, the optimal 
allocation, and the associated probability, will both vary. In particular, increasing the standard deviation of the survival management action will decrease the amount allocated to survival when diversifying allocation in both cases studied (see Supporting Information for analytical justification). In the case of the management of the koala population, such an increase will also delay the transition to allocating the entire budget to managing fecundity for high levels of the threshold. The change in the optimal allocation is given by an analytical expression that we have explicitly derived (Supporting Information equation $\mathrm{S} 1.26)$.

When uncertainties are represented by the uniform distributions (Figs. 2 and 4), all allocations satisfy the objective with probability 1 for values of $\rho$ below the minimum marginal efficiency (that is, the lower bound of the uniform distribution) of both actions,. Then, for $\rho$ values between the minimum marginal efficiency of the two actions, a range of optimal allocations satisfy the objective with probability 1 . Both of these results are intuitive, if both options guarantee the favourable outcome, then any mixture of them will.

Diversification of allocation occurs under the uniform uncertainties model for $\rho$ values greater than the minimum marginal efficiency of both actions, and below the lesser expected marginal efficiency of either action. In both examples studied, this region is relatively small but could be larger in other cases. For larger values of $\rho$, it is optimal to invest the entire budget in the action offering higher expected marginal efficiency. Finally, for $\rho$ values beyond the greater maximum marginal efficiency of the two actions, all allocations are unsuccessful in satisfying the objective with positive probability.

Some differences in the choice of optimal allocation between the two uncertainty models 
stem from the fact that the normal distribution has unbounded support, while the uniform distribution has bounded support. That is, in the case of the normal distribution, one can have arbitrarily large and small marginal efficiencies with non-zero probabilities. As a result, under the normal assumption, there always exists a unique optimal allocation. On the other hand, under the uniform assumption, there are regions of $\rho$ values for which there exist multiple equally optimal allocations.

In constructing the portfolio, Eq. (1), we assumed that the marginal efficiency of the portfolio is a linear combination of the marginal efficiencies of each strategy. Furthermore, by considering only the fraction of the budget allocated to each strategy, a second assumption was made that the marginal efficiency scales linearly with respect to the total amount allocated. Both of these assumptions are likely to be incorrect. The dominant eigenvalue is not in general a linear function of the underlying management parameters. In fact, it is not even an everywhere analytic function of these parameters. We therefore tested the sensitivity of our approach to these two assumptions (see Supporting Information). In particular, we demonstrated how a similar analysis could be conducted in the absence of these assumptions, and applied the resulting procedure to the koala population management case study. Our sensitivity analysis suggested that these assumptions are reasonable for budgets of the order of treating $10 \%$ of the population.

In order to address the assumption of linearly scaling marginal efficiency with respect to the total budget allocated, the decision variables must be changed from fraction allocated to actual budget spent. In accordance, the corresponding constraint would change to ensure the total allocated equals the budget available. This reformulation of the problem would also accommodate stochastic cost functions that are dependent on the actual dollars allocated. This is useful, for example, in the case when potential management actions 
have fixed initial costs. Such fixed costs can then be represented as constraints on the decision variables denoting dollars allocated to each action.

\section{Discussion}

Uncertainty is common in environmental management problems(e.g., Moore et al. 2004) and must be addressed when making decisions. Failure to do so runs the risk of over-confidence in the chosen strategy, and exposure to higher rates of failure (Burgman 2005). Using the case studies for the management of the koala population and the conservation of the olive ridley sea turtle population, we have demonstrated a method to account for uncertainty in population management decisions.

Combining perturbation analysis of matrix population models and safety first portfolio formulation, our results illustrate how one is able to explore the entire decision map of trade-offs between the planned effect on the population per dollar spent, and the probability of achieving it.

Baxter et al. (2006) noted that their results were highly sensitive to the choice of the expected cost point estimates. Our method addresses such sensitivities in two steps. First, portfolios of the actions define the probability of achieving a threshold of acceptability, determined by the assumptions about the uncertainty. Second, an optimisation problem is solved to find the optimal allocation that maximises the probability of obtaining an outcome above the minimally-acceptable value.

From our analyses, diversification of allocation is optimal only for minimally-acceptable 
outcomes below the expected marginal efficiencies of all strategies. Once the level of the minimally-acceptable threshold is above the lower of the two expected marginal efficiencies, it is optimal to allocate the entire budget to only one of the strategies. In other words, for low aspirations, hedging ones bets by diversifying the allocation is the optimal solution. Conversely, for high aspirations, effort is directed solely at management actions that provide the highest potential effect on the population. These results agree with common practice and previous studies (e.g., McCarthy et al. 2010).

The method we propose necessitates making two important choices. First, one must choose an appropriate uncertainty model. In this paper, for illustrative purposes, we use the normal and uniform uncertainty models. In some cases, however, other uncertainty models may be more appropriate. Specifically, our results show that, while some overarching patterns in the optimal allocation exist across models, the optimal allocation is, indeed, sensitive to the choice of the uncertainty model. This fact highlights the importance of characterizing any uncertainty present in the decision process. Characterization of uncertainty could be achieved in one of several ways. In some cases, the uncertainty model can be determined from physical considerations or first principles, alone. In other cases, the uncertainty model can be estimated from historical data. Expert elicitation is another common method for estimating uncertainty models (Garthwaite et al. 2005). Whatever uncertainty model is used, it must be reassessed and updated accordingly if new information becomes available.

The second choice to be considered relates to the value of the threshold of acceptability. This is chosen by considering either the level of confidence (equivalently, the level of risk) one is willing to accept, or the minimum level of efficiency required to satisfy a management goal. The choice is made either a priori, or a posteriori. An a priori 
threshold of acceptability is determined from either physical considerations, previous data, or set rules and regulations. When an a priori choice is not feasible, the threshold of acceptabilitymust be determined a posteriori by examining decision maps such as in Fig. 1-4, and studying the tradeoff between aspiring for higher outcomes and the probability of achieving them.

The safety first portfolio formulation presented here combines ideas of optimal expected utility (Savage 1954), robust optimization (Ghaoui et al. 2009) and satisficing objective functions (Simon 1982). Modified slightly, our approach can also represent other robust decision objectives. For example, constraining the probability of satisfying the threshold of acceptability to unity, and maximizing the threshold of acceptability for a uniform uncertainty model, is equivalent to maximizing the worst-case strategy (French 1986).

While the work presented here concentrated on uncertainty in costs, it could equally be applicable to uncertainties in the population projection matrix estimates. For example, Deines et al. (2007) introduced a method for accounting for uncertainties in the underlying vital rates of the population projection matrix, when using structured population models. Embedding their results in our proposed framework, combined with distributional assumptions for the uncertainty models, extends the safety-first portfolio model that we introduced here to cases where there is uncertainty in other parameters. Furthermore, assuming independence between the uncertainties in cost and vital rates estimates, the two uncertainty models can easily be combined.

The work presented here concentrates on uncertainty in cost estimates, continuous linear cost functions, long-term asymptotic dynamics, and point decisions. Possible future directions include: accounting for explicit uncertainty in population matrix parameters 
using the transfer function (Hodgson \& Townley 2004), extensions to non-linear and non-continuous cost functions, short-term transient dynamics (Caswell 2007), and consecutive decisions.

\section{Acknowledgement}

We thank the reviewers and the handling editor for their insightful comments. This work was supported by the Applied Environmental Decision Analysis research hub that is funded by the Australian Commonwealth Environment Research Facilities program, the Australian Research Council Centre of Excellence for Mathematics and Statistics of Complex Systems and Australian Research Council Discovery Grant DP110101929. M.M.C. and B.W. are also supported by the Australian Research Council Centre of Excellence for Environmental Decisions and Future Fellowships.

\section{Supporting Information}

Detailed derivations for the case of normally (Appendix S1) and uniformly (Appendix S2) distributed uncertainties, as well as derivation of marginal cost expressions (Appendix S3) and analysis of robustness to the linearity assumption (Appendix S4) are all available online. The authors are solely responsible for the content and functionality of these materials. Queries (other than absence of the material) should be directed to the corresponding author. 


\section{References}

Baxter, P. W., M. A. McCarthy, H. P. Possingham, P. W. Menkhorst and N. Mclean, 2006. Accounting for management costs in sensitivity analyses of matrix population models. Conservation Biology 20:893-905.

Bertsekas, D. P., 1999. Nonlinear programming. 2nd edition. Athena Scientific.

Bode, M., K. A. Wilson, T. M. Brooks, W. R. Turner, R. A. Mittermeier, M. F. McBride, E. C. Underwood and H. P. Possingham, 2008. Cost-effective global conservation spending is robust to taxonomic group. Proceedings of the National Academy of Sciences of the United States of America 105:6498-6501.

Burgman, M., 2005. Risks and decisions for conservation and environmental management. Cambridge University Press, Cambridge, UK.

Caswell, H., 2001. Matrix population models: construction, analysis, and interpretation. Sinauer Associates. Sunderland, Massachusetts, USA .

Caswell, H., 2007. Sensitivity analysis of transient population dynamics. Ecology Letters 10:1-15.

Crouse, D., L. Crowder and H. Caswell, 1987. A stage-based population model for loggerhead sea turtles and implications for conservation. Ecology 68:1412-1423.

Crouse, D. T., M. Donnelly, M. J. Bean, A. Clark, W. R. Irvin and C. E. Williams, 1992. The TED experience: claims and reality. Marine Turtle Newsletter 58:6-7.

Crowder, L., D. Crouse and S. Heppell, 1994. Predicting the impact of turtle excluder devices on loggerhead sea turtle populations. Ecological Applications 4:437-445. 
Deines, A., E. Peterson, D. Boeckner, J. Boyle, A. Keighley, J. Kogut, J. Lubben, R. Rebarber, R. Ryan and B. Tenhumberg, 2007. Robust population management under uncertainty for structured population models. Ecological applications : a publication of the Ecological Society of America 17(8):2175-2183.

Edwards, S. F., J. S. Link and B. P. Rountree, 2004. Portfolio management of wild fish stocks. Ecological Economics 49:317-329.

French, S., 1986. Decision theory: an introduction to the mathematics of rationality. Horwood, Chichester, UK.

Garthwaite, P. H., J. B. Kadane and A. O'Hagan, 2005. Statistical method for eliciting probability distributions. Journal of the American Statistical Association 100:680-700.

Ghaoui, E. L., A. Ben-Tal and A. Nemirovski, 2009. Robust Optimization. Princeton University Press, Princeton.

Harbutt, K., 2004. How much can a koala bear? The Weekend Australian Magazine May 8-9:18-21.

Hodgson, D. and S. Townley, 2004. Methodological insight: Linking management changes to population dynamic responses: the transfer function of a projection matrix perturbation. Journal of Applied Ecology 41:1155-1161.

Joseph, L. N., R. F. Maloney and H. P. Possingham, 2009. Optimal Allocation of Resources among Threatened Species: a Project Prioritization Protocol. Conservation Biology 23:328-338.

Markowitz, H., 1952. Portfolio selection. Journal of Finance 7:77-91. 
Markowitz, H., 1991. Foundations of Portfolio Theory. Journal of Finance 46:469-477.

Martin, T. G., I. Chadès, P. Arcese, P. P. Marra, H. P. Possingham and D. R. Norris, 2007. Optimal conservation of migratory species. PLoS ONE 2:e751.

McCarthy, M. A., C. J. Thompson and S. T. Garnett, 2008. Optimal investment in conservation of species. Journal of Applied Ecology 45:1428-1435.

McCarthy, M. A., C. J. Thompson, C. Hauser, M. A. Burgman, H. P. Possingham, M. L. Moir, T. Tiensin and M. Gilbert, 2010. Resource allocation for efficient environmental management. Ecology Letters 13:1280-1289.

McCarthy, M. A., C. J. Thompson and H. P. Possingham, 2005. Theory for designing nature reserves for single species. The American Naturalist 165(2):250-257.

McNeil, A. J., R. Frey and P. Embrechts, 2005. Quantitative risk management. concepts, techniques and tools, Princeton University Press.

Middleton, D. R., B. Walters, P. Menkhorst and P. Wright, 2003. Fertility control in the koala, Phascolarctos cinereus: the impact of slow-release implants containing levonorgestrel or oestradiol on the production of pouch young. Wildlife Research 30:207-212.

Moore, J., A. Balmford, T. Allnutt and N. Burgess, 2004. Integrating costs into conservation planning across Africa. Biological Conservation 117:343-350.

Possingham, H., S. Andelman, B. Noon, S. Trombulak and H. Pulliam, 2001. Making smart conservation decisions. In G. Orians \& M. Soule, editors, Conservation Biology: Research Priorities for the Next Decade, pages 225-244. Island Press, Washington, DC. 
Roy, A., 1952. Safety first and the holding of assets. Econometrica 20:431-449.

Savage, L., 1954. The foundations of statistics. Wiley, New York.

Seminoff, J. and K. Shanker, 2008. Marine turtles and IUCN Red Listing: A review of the process, the pitfalls, and novel assessment approaches. Journal of Experimental Marine Biology and Ecology 356:52-68.

Shanker, K., 1994. Conservation of sea turtles on the Madras coast. Marine Turtle Newsletter 64:3-6.

Simon, H. A., 1982. Models of bounded rationality. MIT Press, Cambridge, MA.

Springer, U., 2003. International diversification of investments in climate change mitigation. Ecological Economics 46:181-193.

Wilson, K. A., M. F. McBride, M. Bode and H. P. Possingham, 2006. Prioritizing global conservation efforts. Nature 440:337-340. 
Table 1: Stage-based population projection matrix for the koala population on Snake Island, Victoria, Australia (data from McLean 2003). The different stages were determined based on tooth wear.

\begin{tabular}{c|ccccccccc}
\hline \hline & \multicolumn{10}{|c}{ life stage at time $t$} \\
time $t+1$ & 1 & 2 & 3 & 4 & 5 & 6 & 7 & 8 & 9 \\
\hline 1 & 0 & 0.3026 & 0.1663 & 0.1244 & 0.0891 & 0.0556 & 0.0394 & 0.0226 & 0.0118 \\
2 & 0.9908 & 0.5359 & 0 & 0 & 0 & 0 & 0 & 0 & 0 \\
3 & 0 & 0.4580 & 0.4550 & 0 & 0 & 0 & 0 & 0 & 0 \\
4 & 0 & 0 & 0.5000 & 0.0655 & 0 & 0 & 0 & 0 & 0 \\
5 & 0 & 0 & 0 & 0.7272 & 0.2216 & 0 & 0 & 0 & 0 \\
6 & 0 & 0 & 0 & 0 & 0.4617 & 0.2265 & 0 & 0 & 0 \\
7 & 0 & 0 & 0 & 0 & 0 & 0.3538 & 0.1267 & 0 & 0 \\
8 & 0 & 0 & 0 & 0 & 0 & 0 & 0.4693 & 0.4247 & 0 \\
9 & 0 & 0 & 0 & 0 & 0 & 0 & 0 & 0.1762 & 0.6090 \\
\hline
\end{tabular}

Table 2: Stage-based population projection matrix for loggerhead sea turtles (Caretta caretta) (data from Crowder et al., 1994). The five stages are: hatchlings/juveniles, small juveniles, large juveniles, subadults, adults. The different stages were determined based on growth rates, age at first reproduction, and survivorship.

\begin{tabular}{c|ccccc}
\hline \hline & \multicolumn{5}{|c}{ life stage at time $t$} \\
time $t+1$ & 1 & 2 & 3 & 4 & 5 \\
\hline 1 & 0 & 0 & 0 & 4.665 & 61.896 \\
2 & 0.675 & 0.703 & 0 & 0 & 0 \\
3 & 0 & 0.047 & 0.657 & 0 & 0 \\
4 & 0 & 0 & 0.019 & 0.682 & 0 \\
5 & 0 & 0 & 0 & 0.061 & 0.809 \\
\hline
\end{tabular}


Figure 1: Plot of optimal allocation for the koala population management example (a) and associated probability of satisfying the minimally-acceptable threshold $\rho(\mathrm{b})$, for the case of normally distributed uncertainties. (a) The dashed and dotted lines denote the expected marginal efficiencies for the contraceptive and translocation strategies respectively. The solid line denotes the optimal fraction of the budget allocated to contraceptive; (b) The y-axis denotes the probability of satisfying the minimally-acceptable threshold. Each curve represents such probability for a different assumption of percentile over the interval in marginal efficiency defined by the estimated per individual costs. The proportional difference in degree of uncertainty between the two management options, $\alpha$, is kept constant across all cases. programs

Figure 2: Plot of optimal allocation for the koala population management example (a) and associated probability of satisfying the minimally-acceptable threshold $\rho(\mathrm{b})$, for the case of uniformly distributed uncertainties. (a) The dashed and dotted lines denote the minimum, expected and maximum marginal efficiencies for the contraceptive and translocation strategies respectively. The solid line denotes the optimal fraction of the budget allocated to contraception, and the shaded area denote the set of all equivalently optimal allocations; (b) The y-axis denotes the probability of satisfying the minimally-acceptable threshold.

Figure 3: Plot of optimal allocation for the sea turtle population management example (a) and associated probability of satisfying the minimally-acceptable threshold $\rho$ (b), for the case of normally distributed uncertainties. (a) The dotted and dashed lines denote the expected marginal efficiencies for the TEDs inclusion and habitat protection strategies respectively. The solid line denotes the optimal fraction of the 
budget allocated to habitat protection; (b) The y-axis denotes the probability of satisfying the minimally-acceptable threshold. Each curve represents such probability for a different assumption of percentile over the interval in marginal efficiency defined by the estimated per individual costs. The proportional difference in degree of uncertainty between the two management options, $\alpha$, is kept constant across all cases.

Figure 4: Plot of optimal allocation for the sea turtle population management example (a) and associated probability of satisfying the minimally-acceptable threshold $\rho$ (b), for the case of uniformly distributed uncertainties. (a) The dotted and dashed lines denote the minimum, and expected marginal efficiencies for the TEDs inclusion, and the minimum marginal efficiency for the habitat protection strategies, respectively. The solid line denotes the optimal fraction of the budget allocated to habitat protection, and the shaded area denote the set of all equivalently optimal allocations; (b) The y-axis denotes the probability of satisfying the minimally-acceptable threshold. 


\section{Supporting Information}

\section{Appendix S1: Normally distributed uncertainties}

\section{Lemmas and Propositions}

Lemma 1.1. Assume the marginal efficiencies for each strategy follow a normal distribution, then the portfolio marginal efficiency $R(\mathbf{x})$, defined in Eq. (1), also follows a normal distribution with mean $E[R(\mathbf{x})]$ and variance $\operatorname{Var}[R(\mathbf{x})]$. The following equivalence holds,

$$
\arg \min _{\mathbf{x}} P\{R(\mathbf{x}) \leq \rho\} \Longleftrightarrow \arg \max _{\mathbf{x}} \frac{(E[R(\mathbf{x})]-\rho)}{\sqrt{\operatorname{Var}[R(\mathbf{x})]}}
$$

Proof. The probability that the marginal efficiency of the portfolio is below a critical value $\rho$ is given by,

$$
\begin{aligned}
P\{R(\mathbf{x}) \leq \rho\} & =P\left\{Z \leq \frac{\rho-E[R(\mathbf{x})]}{\sqrt{\operatorname{Var}[R(\mathbf{x})]}}\right\} \\
& =\Phi\left(\frac{\rho-E[R(\mathbf{x})]}{\sqrt{\operatorname{Var}[R(\mathbf{x})]}}\right) \\
& =\frac{1}{\sqrt{2 \pi}} \int_{-\infty}^{\frac{\rho-E[R(\mathbf{x})]}{\sqrt{\operatorname{Var}[R(\mathbf{x})]}}} e^{-\frac{u^{2}}{2}} d u,
\end{aligned}
$$

where $Z \sim N(0,1)$ is a standard normal random variable, and $\Phi(z)$ its distribution function. Now, since the integrand in Eq. (S1.2) is positive, it is clear that $\Phi(z)$ is a monotonically increasing function of $z$, and the equivalence Eq. (S1.1) follows immediately.

A generalization of the result from Lemma 1.1 to the case where the marginal efficiency of the portfolio follows other distributions apart from normal, was suggested by (Roy, 1952). In 
particular, suppose we are only given the first and second moments of the marginal portfolio efficiency, and no details regarding its explicit distribution. Under such information it is, of course, impossible to determine precisely the probability of the marginal efficiency of the portfolio being less than or equal to some threshold. However, it is still possible to calculate an upper bound on such probability using Chebyshev's inequality.

Lemma 1.2. Suppose we are only given the values $E[R(\mathbf{x})]$ and $\operatorname{Var}[R(\mathbf{x})]$, under all possible allocations $\mathbf{x}$. The probability of $R(\mathbf{x})$ being less than or equal to some threshold $\rho$ has the following property:

$$
P\{R(\mathbf{x}) \leq \rho\} \leq \frac{\operatorname{Var}[R(\mathbf{x})]}{(E[R(\mathbf{x})]-\rho)^{2}} .
$$

Then, obtaining the optimal allocation, $\hat{\mathbf{x}}$, given by

$$
\arg \max _{\mathbf{x}} \frac{(E[R(\mathbf{x})]-\rho)}{\sqrt{\operatorname{Var}[R(\mathbf{x})]}}
$$

is equivalent to finding the allocation that minimises the Chebyshev upper bound on $P\{R(\mathbf{x}) \leq \rho\}$ w.r.t. $\mathbf{x}$.

Proof. Using Chebyshev's inequality we have,

$$
P\{|R(\mathbf{x})-E[R(\mathbf{x})]| \geq(E[R(\mathbf{x})]-\rho)\} \leq \frac{\operatorname{Var}[R(\mathbf{x})]}{(E[R(\mathbf{x})]-\rho)^{2}}
$$

Then, we can rewrite Eq. (S1.5) as,

$$
P\{(E[R(\mathbf{x})]-R(\mathbf{x})) \geq(E[R(\mathbf{x})]-\rho)\}=P\{R(\mathbf{x}) \leq \rho\} \leq \frac{\operatorname{Var}[R(\mathbf{x})]}{(E[R(\mathbf{x})]-\rho)^{2}}
$$

for $\rho<E[R(\mathbf{x})]$, or

$$
P\{(E[R(\mathbf{x})]-R(\mathbf{x})) \geq(\rho-E[R(\mathbf{x})])\}=P\{R(\mathbf{x}) \geq \rho\} \leq \frac{\operatorname{Var}[R(\mathbf{x})]}{(E[R(\mathbf{x})]-\rho)^{2}}
$$


for $\rho \geq E[R(\mathbf{x})]$.

\section{Problem formulation and analytic solutions}

We consider the case where one must decide how to allocate a budget between two potential conservation actions, as is the case with both studies discussed in this paper. The following formulation uses the notation from the koala management example; for the marine turtle example simply replace $f$ by $h$.

We assume that the marginal efficiency for each strategy follows a normal distribution. Then the marginal efficiency of the portfolio, given by Eq. (8), also follows a normal distribution with mean and variance given by,

$$
\begin{gathered}
E[R(x)]=x E\left[R_{f}\right]+(1-x) E\left[R_{s}\right], \\
\operatorname{Var}[R(x)]=x^{2} \operatorname{Var}\left[R_{f}\right]+(1-x)^{2} \operatorname{Var}\left[R_{s}\right] .
\end{gathered}
$$

We are interested in the optimal allocation $(\hat{x})$ which minimises the probability of obtaining a marginal efficiency below a critical value $\rho$. For the normal distribution, this is equivalent to maximising the number of standard deviations $\rho$ is below the expected portfolio marginal efficiency (see Lemma 1.1). The problem $P$ is therefore to find

$$
\begin{aligned}
& \arg \max _{x} \Psi(x)=\frac{E[R(x)]-\rho}{\sqrt{\operatorname{Var}[R(x)]}}=\frac{x E\left[R_{f}\right]+(1-x) E\left[R_{s}\right]-\rho}{\sqrt{x^{2} \operatorname{Var}\left[R_{f}\right]+(1-x)^{2} \operatorname{Var}\left[R_{s}\right]}}, \\
& \text { s.t } 0 \leq x \leq 1,
\end{aligned}
$$


with a Lagrangian given by

$$
L(x, \mathbf{u})=\Psi(x)+u_{1} g_{1}(x)+u_{2} g_{2}(x)=\frac{x E\left[R_{f}\right]+(1-x) E\left[R_{s}\right]-\rho}{\sqrt{x^{2} \operatorname{Var}\left[R_{f}\right]+(1-x)^{2} \operatorname{Var}\left[R_{s}\right]}}+u_{1}(x-1)-u_{2} x
$$

where $u_{1}$ and $u_{2}$ are the Lagrange multipliers of the constraints $g_{1}(x)=x-1 \leq 0$ and $g_{2}(x)=-x \leq 0$, respectively. $P$ is a non-linear constrained optimization problem in the single variable $x$. It could be solved using elementary calculus. However, since we wish to describe a general approach applicable to higher dimension problems (that is, portfolios with more than two alternatives), we solve the problem using the Karush-Kuhn-Tucker (KKT) conditions (Bertsekas 1995). The KKT conditions for the problem described in Eq. (S1.12) are

$$
\begin{gathered}
\nabla_{x} L(\hat{x}, \hat{\mathbf{u}})=0 \\
\hat{u}_{i} \geq 0, \quad i \in\{1,2\} \\
\hat{u}_{i}=0, \quad \forall i \notin A(\hat{x}),
\end{gathered}
$$

where $A(\hat{x})$ is the set of active constraints at $\hat{x}, A(\hat{x})=\left\{i \mid g_{i}(x)=0\right\}$. We note that any optimal solution to the problem must satisfy conditions (S1.13)-(S1.15), and as such they are necessary. If, in addition, the objective function, $\Psi$, and constraints $g_{i}$, are twice continuously differentiable, these conditions become sufficient as well when the objective function is concave (note that the constraints in this case are convex). In particular, we examine three cases:

Case 1: $\rho \leq \min \left\{E\left[R_{f}\right], E\left[R_{s}\right]\right\}$

In this case the solution to eqns. (S1.13)-(S1.15) is

$$
\hat{x}=\frac{\operatorname{Var}\left[R_{s}\right]\left(E\left[R_{f}\right]-\rho\right)}{\operatorname{Var}\left[R_{s}\right]\left(E\left[R_{f}\right]-\rho\right)+\operatorname{Var}\left[R_{f}\right]\left(E\left[R_{s}\right]-\rho\right)},
$$


and $\hat{u}_{1}=\hat{u}_{2}=0$, that is both constraints are inactive.

To show that $\hat{x}$ above is indeed the optimal solution, we first obtain an expression for the critical points of the objective function by solving $d \Psi / d x=0$ where $\Psi(x)$ is given by Eq. (S1.10). We then have

$$
\frac{d \Psi}{d x}=\frac{x \operatorname{Var}\left[R_{f}\right]\left(\rho-E\left[R_{s}\right]\right)-(1-x) \operatorname{Var}\left[R_{s}\right]\left(\rho-E\left[R_{f}\right]\right)}{\left(x^{2} \operatorname{Var}\left[R_{f}\right]+(1-x)^{2} \operatorname{Var}\left[R_{s}\right]\right)^{3 / 2}}=0 .
$$

Solving Eq. (S1.17) for $x$ we obtain Eq. (S1.16). We note that from Eq. (S1.16) there exists only one critical point and that for $\rho \leq \min \left\{E\left[R_{f}\right], E\left[R_{s}\right]\right\}$, the critical point is indeed in $[0,1]$. Furthermore, we have

$$
\left.\frac{d^{2} \Psi}{d x^{2}}\right|_{\hat{x}}=\frac{\operatorname{Var}\left[R_{f}\right]\left(\rho-E\left[R_{s}\right]\right)+\operatorname{Var}\left[R_{s}\right]\left(\rho-E\left[R_{f}\right]\right)}{\left(\hat{x}^{2} \operatorname{Var}\left[R_{f}\right]+(1-\hat{x})^{2} \operatorname{Var}\left[R_{s}\right]\right)^{3 / 2}}<0,
$$

since $\hat{x} \in[0,1]$ and the term in the denominator is always positive while the term in the numerator is negative for $\rho \leq \min \left\{E\left[R_{f}\right], E\left[R_{s}\right]\right\}$. Then, from Eq. (S1.18), the objective function is concave and conditions (S1.13)-(S1.15) become sufficient. We conclude that solution Eq. (S1.16) satisfies both the necessary and sufficient conditions and thus is indeed the global maximum of the objective function Eq. (S1.10), with the Lagrange multipliers $\hat{u}_{1}=\hat{u}_{2}=0$.

Case 2: $\min \left\{E\left[R_{f}\right], E\left[R_{s}\right]\right\} \leq \rho<\max \left\{E\left[R_{f}\right], E\left[R_{s}\right]\right\}$

In this case we examine two distinct events, $E\left[R_{f}\right]<E\left[R_{s}\right]$ and $E\left[R_{f}\right]>E\left[R_{s}\right]$ (note that in the event $E\left[R_{f}\right]=E\left[R_{s}\right]$ case 2 is empty). The solutions to conditions (S1.13)-(S1.15) are

$$
\begin{aligned}
& \hat{x}=0, \quad \hat{u}_{1}=\left(\rho-E\left[R_{f}\right]\right) / \sqrt{\operatorname{Var}\left[R_{s}\right]}, \quad \hat{u}_{2}=0 \quad \text { for } E\left[R_{f}\right]<E\left[R_{s}\right], \\
& \hat{x}=1, \quad \hat{u}_{1}=0, \quad \hat{u}_{2}=\left(\rho-E\left[R_{s}\right]\right) / \sqrt{\operatorname{Var}\left[R_{f}\right]} \quad \text { for } E\left[R_{f}\right]>E\left[R_{s}\right],
\end{aligned}
$$


To show that eqns. (S1.19) and (S1.20) indeed satisfy conditions (S1.13)-(S1.15) we observe that for

$$
\min \left\{E\left[R_{f}\right], E\left[R_{s}\right]\right\} \leq \rho<\max \left\{E\left[R_{f}\right], E\left[R_{s}\right]\right\}
$$

and $x \in[0,1], d \Psi / d x \neq 0$ and $d^{2} \Psi /\left.d x^{2}\right|_{\hat{x}}<0$. We therefore conclude that the function is concave and monotone. Then substituting $\hat{x}=0$ and $\hat{x}=1$ in Eq. (S1.13) we have for $E\left[R_{f}\right] \leq \rho \leq E\left[R_{s}\right]$,

$$
\hat{u}_{1}=\left(\rho-E\left[R_{f}\right]\right) / \sqrt{\operatorname{Var}\left[R_{s}\right]}>0, \quad \hat{u}_{2}=\left(\rho-E\left[R_{s}\right]\right) / \sqrt{\operatorname{Var}\left[R_{f}\right]}<0
$$

and for $E\left[R_{s}\right] \leq \rho \leq E\left[R_{f}\right]$,

$$
\hat{u}_{1}=\left(\rho-E\left[R_{f}\right]\right) / \sqrt{\operatorname{Var}\left[R_{s}\right]}<0, \quad \hat{u}_{2}=\left(\rho-E\left[R_{s}\right]\right) / \sqrt{\operatorname{Var}\left[R_{f}\right]}>0 .
$$

Case 3: $\rho \geq \max \left\{E\left[R_{f}\right], E\left[R_{s}\right]\right\}$

Here, again as in case 2, we examine two distinct events, $\left.\left(E\left[R_{s}\right]-\rho\right) / \sqrt{\operatorname{Var}\left[R_{s}\right]}>\left(E\left[R_{f}\right]-\rho\right) / \sqrt{\operatorname{Var}\left[R_{f}\right.}\right]$ and $\left(E\left[R_{s}\right]-\rho\right) / \sqrt{\operatorname{Var}\left[R_{s}\right]}<\left(E\left[R_{f}\right]-\rho\right) / \sqrt{\operatorname{Var}\left[R_{f}\right]}$. The solutions to conditions (S1.13)-(S1.15) are

$$
\begin{array}{r}
\hat{x}=0, \quad \hat{u}_{1}=0, \quad \hat{u}_{2}=\left(E\left[R_{f}\right]-\rho\right) / \sqrt{\operatorname{Var}\left[R_{s}\right]} \\
\text { for } \quad\left(E\left[R_{s}\right]-\rho\right) / \sqrt{\operatorname{Var}\left[R_{s}\right]}>\left(E\left[R_{f}\right]-\rho\right) / \sqrt{\operatorname{Var}\left[R_{f}\right]}
\end{array}
$$

and,

$$
\begin{gathered}
\hat{x}=1, \quad \hat{u}_{1}=\left(\rho-E\left[R_{s}\right]\right) / \sqrt{\operatorname{Var}\left[R_{f}\right]}, \quad \hat{u}_{2}=0 \\
\text { for } \quad\left(E\left[R_{s}\right]-\rho\right) / \sqrt{\operatorname{Var}\left[R_{s}\right]}<\left(E\left[R_{f}\right]-\rho\right) / \sqrt{\operatorname{Var}\left[R_{f}\right]}
\end{gathered}
$$


To show that eqns. (S1.23) and (S1.24) indeed satisfy conditions eqns. (S1.13)-(S1.15) we first observe that for $\rho \geq \max \left\{E\left[R_{f}\right], E\left[R_{s}\right]\right\}$ and $\hat{x}$ given by Eq. (S1.16), $d^{2} \Psi /\left.d x^{2}\right|_{\hat{x}}>0$. We therefore conclude that the objective function is convex and thus the boundary solutions are local maxima and we proceed by comparing the value of the objective function for each such solution.

So far the analysis of the normal distribution has assumed that the variance for each of the management programs, $\operatorname{Var}\left[R_{f}\right]$ and $\operatorname{Var}\left[R_{s}\right]$, is given. However, as mentioned earlier, these variances are a function of the assumed percentage of the distribution centred around the mid point of the range. In other words, the variances represent some measure of the degree of our uncertainty regarding the estimate of the marginal efficiency for each management program. To better indicate the dependence of the optimal allocation on the discrepancy in our uncertainty with regards to each program we let

$$
\sqrt{\operatorname{Var}\left[R_{f}\right]}=\alpha \sqrt{\operatorname{Var}\left[R_{s}\right]}
$$

where $\alpha$ represents the proportional difference in degree of uncertainty between the two management programs. Then, summarising the three cases using the relationship in Eq. (S1.25), the optimal 
allocation is given by

$$
\hat{x}=\left\{\begin{aligned}
\frac{\left(E\left[R_{f}\right]-\rho\right)}{\left(E\left[R_{f}\right]-\rho\right)+\alpha^{2}\left(E\left[R_{s}\right]-\rho\right)} \quad \text { for } 0 \leq \rho \leq \min \left\{E\left[R_{f}\right], E\left[R_{s}\right]\right\} \\
1 \quad \text { for } \min \left\{E\left[R_{f}\right], E\left[R_{s}\right]\right\}<\rho \leq \max \left\{E\left[R_{f}\right], E\left[R_{s}\right]\right\} \\
\quad \text { and } E\left[R_{f}\right]>E\left[R_{s}\right], \\
\text { or } \rho \geq \max \left\{E\left[R_{f}\right], E\left[R_{s}\right]\right\} \\
\quad \text { and }\left(E\left[R_{s}\right]-\rho\right) \alpha<\left(E\left[R_{f}\right]-\rho\right), \\
\text { for } \min \left\{E\left[R_{f}\right], E\left[R_{s}\right]\right\}<\rho \leq \max \left\{E\left[R_{f}\right], E\left[R_{s}\right]\right\} \\
\quad \text { and } E\left[R_{f}\right]<E\left[R_{s}\right], \\
\text { or } \rho \geq \max \left\{E\left[R_{f}\right], E\left[R_{s}\right]\right\} \\
\quad \text { and }\left(E\left[R_{s}\right]-\rho\right) \alpha>\left(E\left[R_{f}\right]-\rho\right)
\end{aligned}\right.
$$

where the allocation is a function only of the expected marginal efficiency of each management program, the critical value $\rho$ and $\alpha$. Also note that the optimal allocation does not depend on $\sqrt{\operatorname{Var}\left[R_{f}\right]}$ or $\sqrt{\operatorname{Var}\left[R_{s}\right]}$, only their relative magnitude $\alpha$.

As well as the optimal allocation, our approach also provides the probability of satisfying the critical threshold, $\rho$, for each optimal allocation. Obtaining from Eq. (S1.8)

$$
E(R(\hat{x}))=\hat{x} E\left[R_{f}\right]+(1-\hat{x}) E\left[R_{s}\right]
$$

and from eqns. (S1.28) and (S1.25)

$$
\sigma_{p}^{2}(\hat{x})=\hat{x}^{2} \operatorname{Var}\left[R_{f}\right]+(1-\hat{x})^{2} \operatorname{Var}\left[R_{s}\right]=\operatorname{Var}\left[R_{s}\right]\left(\alpha^{2} \hat{x}^{2}+(1-\hat{x})^{2}\right),
$$

this probability is given by evaluating the normal distribution function for each optimal allocation, 
$\hat{x}$, and critical value, $\rho$,

$$
F(\hat{x}, \rho)=\Phi\left(\frac{\rho-E[R(\hat{x})]}{\sqrt{\operatorname{Var}[R(\hat{x})]}}\right)=\frac{1}{2}\left(1+\operatorname{erf}\left(\frac{\rho-E[R(\hat{x})]}{\sqrt{2 \operatorname{Var}[R(\hat{x})]}}\right)\right)
$$

where $\operatorname{erf}()$ is the error function;

\section{Appendix S2: Uniformly distributed uncertainties}

\section{Lemmas and Propositions}

Lemma 3.3. Let $R_{f} \sim U\left(\tilde{a}_{f}, \tilde{b}_{f}\right)$ and $R_{s} \sim U\left(\tilde{a}_{s}, \tilde{b}_{s}\right)$ be two uniformly distributed random variables, and $R$ also be a random variable defined as the sum of $R_{f}$ and $R_{s}, R=R_{f}+R_{s}$. Then the distribution function for $R$ is given by,

$$
\begin{aligned}
F_{R}(C)= & \int_{-\infty}^{C} \frac{1}{\left(\tilde{b}_{s}-\tilde{a}_{s}\right)\left(\tilde{b}_{f}-\tilde{a}_{f}\right)}\left[\left(w-\tilde{a}_{f}-\tilde{a}_{s}\right) \mathscr{U}\left(w-\tilde{a}_{f}-\tilde{a}_{s}\right)-\left(w-\tilde{a}_{f}-\tilde{b}_{s}\right) \mathscr{U}\left(w-\tilde{a}_{f}-\tilde{b}_{s}\right)\right. \\
& \left.-\left(w-\tilde{b}_{f}-\tilde{a}_{s}\right) \mathscr{U}\left(w-\tilde{b}_{f}-\tilde{a}_{s}\right)+\left(w-\tilde{b}_{f}-\tilde{b}_{s}\right) \mathscr{U}\left(w-\tilde{b}_{f}-\tilde{b}_{s}\right)\right] d w .
\end{aligned}
$$

More specifically, probability, $P\{R \leq C\}$ equals: 
Case 1: $\tilde{a}_{f}+\tilde{b}_{s}<\tilde{a}_{s}+\tilde{b}_{f}$

$$
P\{R \leq C\}= \begin{cases}0 & C<\tilde{a}_{s}+\tilde{a}_{f} \\ \frac{\left(C-\tilde{a}_{s}-\tilde{a}_{f}\right)^{2}}{2\left(\tilde{b}_{s}-\tilde{a}_{s}\right)\left(\tilde{b}_{f}-\tilde{a}_{f}\right)} & \tilde{a}_{s}+\tilde{a}_{f} \leq C<\tilde{a}_{f}+\tilde{b}_{s} \\ \frac{2 C-2 \tilde{a}_{f}-\tilde{b}_{s}-\tilde{a}_{s}}{2\left(\tilde{b}_{f}-\tilde{a}_{f}\right)} & \tilde{a}_{f}+\tilde{b}_{s} \leq C<\tilde{b}_{f}+\tilde{a}_{s} \\ 1-\frac{\left(\tilde{b}_{s}-\tilde{b}_{f}-C\right)^{2}}{2\left(\tilde{b}_{s}-\tilde{a}_{s}\right)\left(\tilde{b}_{f}-\tilde{a}_{f}\right)} & \tilde{a}_{f}+\tilde{b}_{s} \leq C<\tilde{b}_{f}+\tilde{b}_{s} \\ 1 & C>\tilde{b}_{f}+\tilde{b}_{s} .\end{cases}
$$

Case 2: $\tilde{a}_{s}+\tilde{b}_{f}<\tilde{a}_{f}+\tilde{b}_{s}$

$$
P\{R \leq C\}= \begin{cases}0 & C<\tilde{a}_{s}+\tilde{a}_{f} \\ \frac{\left(C-\tilde{a}_{s}-\tilde{a}_{f}\right)^{2}}{2\left(\tilde{b}_{s}-\tilde{a}_{s}\right)\left(\tilde{b}_{f}-\tilde{a}_{f}\right)} & \tilde{a}_{s}+\tilde{a}_{f} \leq C<\tilde{a}_{s}+\tilde{b}_{f} \\ \frac{2 C-2 \tilde{a}_{s}-\tilde{b}_{f}-\tilde{a}_{f}}{2\left(\tilde{b}_{s}-\tilde{a}_{s}\right)} & \tilde{a}_{s}+\tilde{b}_{f} \leq C<\tilde{b}_{s}+\tilde{a}_{f} \\ 1-\frac{\left(\tilde{b}_{s}-\tilde{b}_{f}-C\right)^{2}}{2\left(\tilde{b}_{s}-\tilde{a}_{s}\right)\left(\tilde{b}_{f}-\tilde{a}_{f}\right)} & \tilde{a}_{f}+\tilde{b}_{s} \leq C<\tilde{b}_{f}+\tilde{b}_{s} \\ 1 & C>\tilde{b}_{f}+\tilde{b}_{s} .\end{cases}
$$


Case 3: $\tilde{a}_{s}+\tilde{b}_{f}=\tilde{a}_{f}+\tilde{b}_{s}$

$$
P\{R \leq C\}= \begin{cases}0 & C<\tilde{a}_{s}+\tilde{a}_{f} \\ \frac{\left(C-\tilde{a}_{s}-\tilde{a}_{f}\right)^{2}}{2\left(\tilde{b}_{s}-\tilde{a}_{s}\right)\left(\tilde{b}_{f}-\tilde{a}_{f}\right)} & \tilde{a}_{s}+\tilde{a}_{f} \leq C<\tilde{a}_{s}+\tilde{b}_{f}=\tilde{a}_{f}+\tilde{b}_{s} \\ 1-\frac{\left(\tilde{b}_{s}-\tilde{b}_{f}-C\right)^{2}}{2\left(\tilde{b}_{s}-\tilde{a}_{s}\right)\left(\tilde{b}_{f}-\tilde{a}_{f}\right)} & \tilde{a}_{f}+\tilde{b}_{s}=\tilde{a}_{s}+\tilde{b}_{f} \leq C<\tilde{b}_{f}+\tilde{b}_{s} \\ 1 & C>\tilde{b}_{f}+\tilde{b}_{s} .\end{cases}
$$

Proof - (only included for clarity at this stage. Will be omitted later). The probability density function of the sum of two random variables is given by the convolution of the probability density function of each term.

$$
f_{R}=f_{R_{f}} * f_{R_{s}} .
$$

From convolution theorem we have,

$$
\mathcal{L}\left\{f_{R_{f}} * f_{R_{s}}\right\}=\mathcal{L}\left\{f_{R_{f}}\right\} \mathcal{L}\left\{f_{R_{s}}\right\},
$$

where $\mathcal{L}\{f\}$ denotes the Laplace transform. Writing the probability density function of $R_{f}$ as,

$$
f_{R_{f}}(C)=\frac{I\left(C \in\left[\tilde{a}_{f}, \tilde{b}_{f}\right]\right)}{\tilde{b}_{f}-\tilde{a}_{f}}
$$

then its Laplace transform is given by,

$$
\begin{aligned}
\mathcal{L}\left\{f_{R_{f}}\right\} & =\int_{0}^{\infty} \frac{I\left(C \in\left[\tilde{a}_{f}, \tilde{b}_{f}\right]\right)}{\tilde{b}_{f}-\tilde{a}_{f}} e^{-s C} d C \\
& =\frac{e^{-s \tilde{a}_{f}}-e^{-s \tilde{b}_{f}}}{\left(\tilde{b}_{f}-\tilde{a}_{f}\right) s}
\end{aligned}
$$


where we have assumed $\tilde{a}_{f} \geq 0$. Similarly we have for $R_{s}$,

$$
\mathcal{L}\left\{f_{R_{s}}\right\}=\frac{e^{-s \tilde{a}_{s}}-e^{-s \tilde{b}_{s}}}{\left(\tilde{b}_{s}-\tilde{a}_{s}\right) s},
$$

and thus,

$$
\begin{aligned}
\mathcal{L}\left\{f_{R}\right\} & =\mathcal{L}\left\{f_{R_{f}}\right\} \mathcal{L}\left\{f_{R_{s}}\right\} \\
& =\frac{1}{s^{2}}\left(\frac{e^{-s\left(\tilde{a}_{f}+\tilde{a}_{s}\right)}-e^{-s\left(\tilde{a}_{s}+\tilde{b}_{f}\right)}-e^{-s\left(\tilde{b}_{s}+\tilde{a}_{f}\right)}+e^{-s\left(\tilde{b}_{s}+\tilde{b}_{f}\right)}}{\left(\tilde{b}_{s}-\tilde{a}_{s}\right)\left(\tilde{b}_{f}-\tilde{a}_{f}\right)}\right)
\end{aligned}
$$

Finally, using the second translation theorem we have,

$$
\begin{aligned}
f_{R}(C)= & \mathcal{L}^{-1}\left\{\mathcal{L}\left\{f_{R}\right\}\right\} \\
= & \frac{1}{\left(\tilde{b}_{s}-\tilde{a}_{s}\right)\left(\tilde{b}_{f}-\tilde{a}_{f}\right)}\left[\left(C-\tilde{a}_{f}-\tilde{a}_{s}\right) \mathscr{U}\left(C-\tilde{a}_{f}-\tilde{a}_{s}\right)-\left(C-\tilde{a}_{f}-\tilde{b}_{s}\right) \mathscr{U}\left(C-\tilde{a}_{f}-\tilde{b}_{s}\right)\right. \\
& \left.-\left(C-\tilde{b}_{f}-\tilde{a}_{s}\right) \mathscr{U}\left(C-\tilde{b}_{f}-\tilde{a}_{s}\right)+\left(C-\tilde{b}_{f}-\tilde{b}_{s}\right) \mathscr{U}\left(C-\tilde{b}_{f}-\tilde{b}_{s}\right)\right]
\end{aligned}
$$

where $\mathscr{U}(t-k)=1$, for $t \geq k$, and 0 otherwise, and,

$$
\begin{aligned}
F_{R}(C)= & \int_{-\infty}^{C} f_{R}(w) d w \\
= & \int_{-\infty}^{C} \frac{1}{\left(\tilde{b}_{s}-\tilde{a}_{s}\right)\left(\tilde{b}_{f}-\tilde{a}_{f}\right)}\left[\left(w-\tilde{a}_{f}-\tilde{a}_{s}\right) \mathscr{U}\left(w-\tilde{a}_{f}-\tilde{a}_{s}\right)-\left(w-\tilde{a}_{f}-\tilde{b}_{s}\right) \mathscr{U}\left(w-\tilde{a}_{f}-\tilde{b}_{s}\right)\right. \\
& \left.-\left(w-\tilde{b}_{f}-\tilde{a}_{s}\right) \mathscr{U}\left(w-\tilde{b}_{f}-\tilde{a}_{s}\right)+\left(w-\tilde{b}_{f}-\tilde{b}_{s}\right) \mathscr{U}\left(w-\tilde{b}_{f}-\tilde{b}_{s}\right)\right] d w
\end{aligned}
$$

Returning to the problem of portfolio allocation, we are interested in obtaining an expression for the distribution function of the marginal efficiency of the portfolio $R(x)$, as defined in Eq. (8), 
for the case when $R_{f}$ and $R_{s}$ are uniformly distributed random variables.

Proposition 1. Let $R_{f} \sim U\left(a_{f}, b_{f}\right)$ and $R_{s} \sim U\left(a_{s}, b_{s}\right)$. Then the distribution function of their mixture, $R(x)$, is given by,

$$
\begin{aligned}
F_{R}(C, x)= & \int_{-\infty}^{C} f_{R}(w, x) d w \\
= & \int_{-\infty}^{w} \frac{1}{x(1-x)\left(b_{s}-a_{s}\right)\left(b_{f}-a_{f}\right)}\left[\left(w-x a_{f}-(1-x) a_{s}\right) \mathscr{U}\left(w-x a_{f}-(1-x) a_{s}\right)\right. \\
& -\left(w-x a_{f}-(1-x) b_{s}\right) \mathscr{U}\left(w-x a_{f}-(1-x) b_{s}\right) \\
& -\left(w-x b_{f}-(1-x) a_{s}\right) \mathscr{U}\left(w-x b_{f}-(1-x) a_{s}\right) \\
& \left.+\left(w-x b_{f}-(1-x) b_{s}\right) \mathscr{U}\left(w-x b_{f}-(1-x) b_{s}\right)\right] d w
\end{aligned}
$$

Proof. From Lemma 3.3, substituting $\left\{\tilde{a}_{f}, \tilde{b}_{f}\right\} \rightarrow\left\{x a_{f}, x b_{f}\right\}$ and $\left\{\tilde{a}_{s}, \tilde{b}_{s}\right\} \rightarrow\left\{(1-x) a_{f},(1-x) b_{f}\right\}$ in Eq. (S2.1).

Lemma 3.4. Let $g:(x, C) \rightarrow \mathbb{R}$ be some continuous function on the domain $\mathscr{D}=\{(x, C): x \in$ $(0,1), C \in \mathbb{R}\}$. Then,

$$
u(x, C):=g(x, C)-(C-\alpha x) \mathscr{U}(C-\alpha x), \quad \alpha>C
$$

is continuous with respect to both $x$ and $C$ for all $(x, C) \in \mathscr{D}$.

Proof. We examine the continuity of $u(x, C)$ about the point $C=\alpha x$. In particular,

$$
\lim _{C \rightarrow \alpha x^{+}} u(x, C)=\lim _{C \rightarrow \alpha x^{-}} u(x, C)=g(x, \alpha x),
$$


and,

$$
\lim _{x \rightarrow \frac{C}{\alpha}^{+}} u(x, C)=\lim _{x \rightarrow \frac{C}{\alpha}^{-}} u(x, C)=g\left(\frac{C}{\alpha}, C\right),
$$

where we have used the fact that both $g(x, C)$ and $(C-\alpha x) \mathscr{U}(C-\alpha x)$ are continuous in $\mathscr{D}$.

Lemma 3.5. The probability distribution function Eq. (S2.10) is differentiable with respect to $C$ for all $x \in(0,1)$.

Proof. We first note that the probability density function $f_{R}(x, C)$ is continuous with respect to $C$ for $x \in(0,1)$ using Lemma 3.4 since each one of the four accumulation points of $f_{R}(x, C)$ can be analysed locally as in Lemma 3.4. Then, using the Fundamental Theorem of Calculus, we have,

$$
\begin{aligned}
\frac{\partial}{\partial C} F_{R}(C, x) & =\frac{\partial}{\partial C} \int_{-\infty}^{C} f_{R}(w, x) d w \\
& =f_{R}(C, x) \square
\end{aligned}
$$

It is important to note that the distribution function is not differentiable for the special cases $x=0$ or $x=1$. In these cases the density function $f(x, C)$ collapses to the standard uniform distribution (see Proposition 2 bellow), which is of course discontinuous, and therefore the distribution function is not differentiable.

Lemma 3.6. Let $U:(x, C) \rightarrow \mathbb{R}$ be defined by,

$$
U(x, C):=\int_{-\infty}^{C}(w-\alpha x) \mathscr{U}(w-\alpha x)-(w-\beta x) \mathscr{U}(w-\beta x) d w, \quad \beta>\alpha>C
$$

over the domain $\mathscr{D}=\{(x, C): x \in[0,1], C \in \mathbb{R}\}$. Then $U(x, C)$ is differentiable w.r.t. $x$ for all $x \in[0,1]$. 
Proof. First, $U(x, C)$ is continuous about $x=C / \beta$ since,

$$
\lim _{x \rightarrow \frac{C^{+}}{\beta}} U(x, C)=\lim _{x \rightarrow \frac{C}{\beta}^{-}} U(x, C)=\int_{\alpha x}^{C}(w-\alpha x) d w
$$

Then $U(x, C)$ is differentiable about the point $x=C / \beta$ since,

$$
\begin{aligned}
\lim _{x \rightarrow \frac{C^{+}}{\beta}} \frac{\partial}{\partial x} U(x, C) & =\lim _{x \rightarrow \frac{C}{\beta}} \frac{\partial}{\partial x}\left(\int_{\alpha x}^{C}(w-\alpha x) d w\right) \\
& =\lim _{x \rightarrow \frac{C^{+}}{\beta}} \frac{\partial}{\partial x} \frac{1}{2}(C-\alpha x)^{2} \\
& =\lim _{x \rightarrow \frac{C}{\beta}^{+}}-\alpha(C-\alpha x) \\
& =-\alpha\left(C-\frac{\alpha C}{\beta}\right)
\end{aligned}
$$

and,

$$
\begin{aligned}
\lim _{x \rightarrow \frac{C}{\beta}^{-}} \frac{\partial}{\partial x} U(x, C) & =\lim _{x \rightarrow \frac{C^{-}}{\beta}} \frac{\partial}{\partial x}\left(\int_{\alpha x}^{\beta x}(w-\alpha x) d w+\int_{\beta x}^{C}(w-\alpha x-w+\beta x) d w\right) \\
& =\lim _{x \rightarrow \frac{C^{-}}{\beta}} \frac{\partial}{\partial x} \frac{1}{2}\left(2 C(\beta x-\alpha x)+\alpha^{2} x^{2}-\beta^{2} x^{2}\right) \\
& =\lim _{x \rightarrow \frac{C}{\beta}^{-}}\left(C(\beta-\alpha)+\alpha^{2} x-\beta^{2} x\right) \\
& =-\alpha\left(C-\frac{\alpha C}{\beta}\right)
\end{aligned}
$$

Proposition 2. The distribution function, Eq. (S2.10), is differentiable on $x \in[0,1]$.

Proof. First, the distribution function Eq. (S2.10) is continuous on $x \in[0,1]$, from Lemma 3.6, and since, using L'Hôpital's rule, we have,

$$
\lim _{x \rightarrow 0^{+}} F_{R}(C, x)=\frac{C-a_{s}}{b_{s}-a_{s}},
$$


and,

$$
\lim _{x \rightarrow 1^{-}} F_{R}(C, x)=\frac{C-a_{f}}{b_{f}-a_{f}}
$$

Then, since each one of the four points of the distribution function Eq. (S2.10) can be analyzed locally as in Lemma 3.6 we have our result.

\section{Problem formulation and analytic solutions}

Again, we consider the case where one must decide how to allocate a budget between two potential conservation actions, as per both case studies used for illustration. The following formulation uses the notation from the koala management example; for the marine turtle example simply replace $f$ by $h$.

In this section, we assume the marginal efficiency for each strategy follows a uniform distribution, $R_{f} \sim U\left(a_{f}, b_{f}\right)$ and $R_{s} \sim U\left(a_{s}, b_{s}\right)$. Then the distribution function for the marginal efficiency of the portfolio is given by (see Proposition 1)

$$
\begin{aligned}
P\{R(\mathbf{x}) \leq C\}= & \int_{-\infty}^{C} \frac{1}{x(1-x)\left(b_{s}-a_{s}\right)\left(b_{f}-a_{f}\right)}\left[\left(w-x a_{f}-(1-x) a_{s}\right) \mathscr{U}\left(w-x a_{f}-(1-x) a_{s}\right)\right. \\
& -\left(w-x a_{f}-(1-x) b_{s}\right) \mathscr{U}\left(w-x a_{f}-(1-x) b_{s}\right) \\
& -\left(w-x b_{f}-(1-x) a_{s}\right) \mathscr{U}\left(w-x b_{f}-(1-x) a_{s}\right) \\
& \left.+\left(w-x b_{f}-(1-x) b_{s}\right) \mathscr{U}\left(w-x b_{f}-(1-x) b_{s}\right)\right] d w \\
= & \Psi(x) .
\end{aligned}
$$


where $\mathscr{U}(t-x)$ is a step function, taking the value 1 when $t \geq x$ and 0 otherwise.

We are interested in the allocation, $\hat{x}$, that minimizes the probability of obtaining a marginal efficiency below a critical value $\rho$. The non-linear constraint optimization problem $P$ is therefore

$$
\begin{aligned}
& \arg \min _{x} \Psi(x) \\
& \text { s.t } 0 \leq x \leq 1,
\end{aligned}
$$

with a Lagrangian given by,

$$
L(x, u)=\Psi(x)+u_{1} g_{1}(x)+u_{2} g_{2}(x)=\Psi(x)+u_{1}(x-1)-u_{2} x,
$$

where $u_{1}$ and $u_{2}$ are the Lagrange multipliers of the constraints $g_{1}(x)=x-1 \leq 0$ and $g_{2}(x)=$ $-x \leq 0$, respectively.

Solving $P$ using the KKT necessary conditions eqns. (S1.13)-(S1.14) we obtain expressions for the optimal allocation as function of $\rho$, the minimal marginal efficiency. First we note that the four step function in Eq. (S2.1) divide the space $\{(x, \rho) \mid x \in[0,1], \rho \in \mathbb{R}\}$ into six regions. These six regions change depending on the values of the four parameters $\left(a_{f}, b_{f}\right)$ and $\left(a_{s}, b_{s}\right)$. In each of these six regions the distribution function, Eq. (S2.1), and its partial derivative w.r.t. $x$ assume a different form. These are,

Region 1: $x b_{f}+(1-x) a_{s} \leq \rho \leq x a_{f}+(1-x) b_{s}$

$$
\begin{aligned}
F(x, \rho) & =\frac{2 \rho-x\left(b_{f}+a_{f}\right)-2(1-x) a_{s}}{2(1-x)\left(b_{s}-a_{s}\right)} \\
\frac{\partial F}{\partial x} & =\frac{2 \rho-b_{f}-a_{f}}{2(1-x)^{2}\left(b_{s}-a_{s}\right)}
\end{aligned}
$$


Region 2: $x a_{f}+(1-x) a_{s} \leq \rho \leq\left(x b_{f}+(1-x) a_{s}\right.$ and $\left.x a_{f}+(1-x) b_{s}\right)$

$$
\begin{aligned}
F(x, \rho) & =\frac{\left(\rho-x\left(a_{f}+a_{s}\right)+a_{s}\right)^{2}}{2 x(1-x)\left(b_{s}-a_{s}\right)\left(b_{f}-a_{f}\right)} \\
\frac{\partial F}{\partial x} & =\frac{\left(\rho-x\left(a_{f}+a_{s}\right)+a_{s}\right)\left(2\left(a_{s}-a_{f}\right) x(1-x)-(1-2 x)\left(\rho-x\left(a_{f}+a_{s}\right)+a_{s}\right)\right)}{2 x^{2}(1-x)^{2}\left(b_{s}-a_{s}\right)\left(b_{f}-a_{f}\right)}
\end{aligned}
$$

Region 3: $x a_{f}+(1-x) b_{s} \leq \rho \leq x b_{f}+(1-x) a_{s}$

$$
\begin{aligned}
F(x, \rho) & =\frac{2 \rho-(1-x)\left(b_{s}+a_{s}\right)-2 x a_{f}}{2 x\left(b_{f}-a_{f}\right)} \\
\frac{\partial F}{\partial x} & =\frac{b_{s}+a_{s}-2 \rho}{2 x^{2}\left(b_{f}-a_{f}\right)}
\end{aligned}
$$

Region 4: $\left(x b_{f}+(1-x) a_{s}\right.$ and $\left.x a_{f}+(1-x) b_{s}\right) \leq \rho \leq x b_{f}+(1-x) b_{s}$

$$
\begin{aligned}
F(x, \rho) & =1-\frac{\left(x\left(b_{f}-b_{s}\right)+b_{s}-\rho\right)^{2}}{2 x(1-x)\left(b_{s}-a_{s}\right)\left(b_{f}-a_{f}\right)} \\
\frac{\partial F}{\partial x} & =-\frac{\left(x\left(b_{f}-b_{s}\right)+b_{s}-\rho\right)\left(2\left(b_{f}-b_{s}\right) x(1-x)-(1-2 x)\left(x\left(b_{f}-b_{s}\right)+b_{s}-\rho\right)\right)}{2 x^{2}(1-x)^{2}\left(b_{s}-a_{s}\right)\left(b_{f}-a_{f}\right)}
\end{aligned}
$$

Regions 5: $\rho<x a_{f}+(1-x) a_{s}$

$$
\begin{aligned}
F(x, \rho) & =0 \\
\frac{\partial F}{\partial x} & =0
\end{aligned}
$$


Regions 6: $\rho>x a_{f}+(1-x) a_{s}$

$$
\begin{aligned}
F(x, \rho) & =1 \\
\frac{\partial F}{\partial x} & =0
\end{aligned}
$$

In reporting the expressions for the optimal allocation we distinguish between three different classes of the problem.

- when the ranges of marginal efficiency for each action do not overlap, $a_{f}<b_{f}<a_{s}<b_{s}$,

- when one range is contained within the other, $a_{f}<a_{s}<b_{s}<b_{f}$, and

- when the ranges overlap, $a_{f}<a_{s}<b_{f}<b_{s}$.

Class 1: $a_{f}<b_{f}<a_{s}<b_{s}$

Within this class, we make a further distinction into different cases based on values of $\rho$, the minimal marginal efficiency threshold.

Case 1: $\rho<a_{s}$

In this case the optimal solution is the set of allocations,

$$
\hat{x} \in\left[0, \min \left\{1, \frac{a_{s}-\rho}{a_{s}-a_{f}}\right\}\right]
$$

and $u_{1}=u_{2}=0$. For all values of $x$ in Eq. (S2.37), the probability of the marginal efficiency of the portfolio being below $\rho$ equals zero, $P\{R(\mathbf{x}) \leq \rho\}=0$, which is clearly the minimum.

Case 2: $a_{s} \leq \rho<b_{s}$ 
Here the optimal allocation is, $\hat{x}=0, \hat{u}_{1}=0$, and $\hat{u}_{2}=\left(2 \rho-b_{f}-a_{f}\right) / 2\left(b_{s}-a_{s}\right)$.

Case 3: $\rho>b_{s}$

In this case, for all $x \in[0,1]$ the probability that the portfolio marginal efficiency is below the critical threshold $\rho$ is 1 . In other words, any allocation will fail to satisfy the threshold.

Class 2: $a_{f}<a_{s}<b_{s}<b_{f}$

This class represents the case when one range is contained in the other, as mentioned above. It turns out that the position of the contained range within the containing range also has an effect on the solution to the problem. To assist the reporting of the optimal allocation solutions, we fix the contained range in a region of the containing range. In particular, we set the additional assumptions: $a_{s}<\left(b_{f}+a_{f}\right) / 2<\left(b_{s}+a_{s}\right) / 2$. We report here only the solutions for this special case, while the solutions for the remaining cases could be obtained in similar fashion. The optimal allocation is,

$$
\hat{x}=\left\{\begin{array}{lll}
{\left[0, \min \left\{1, \frac{a_{s}-\rho}{a_{s}-a_{f}}\right\}\right]} & \hat{u}_{1}=\hat{u}_{2}=0 & \text { for } \rho<a_{s} \\
\frac{\rho-a_{s}}{2 \rho-a_{f}-a_{s}} & \hat{u}_{1}=\hat{u}_{2}=0 & \text { for } a_{s} \leq \rho \leq \frac{1}{2}\left(b_{f}+a_{f}\right) \\
0 & \hat{u}_{1}=0, \hat{u}_{2}=\frac{2 \rho-b_{f}-a_{f}}{2\left(b_{s}-a_{s}\right)} & \text { for } \frac{1}{2}\left(b_{f}+a_{f}\right) \leq \rho \leq \frac{a_{s} b_{f}-a_{f} b_{s}}{\left(b_{f}-b_{s}\right)+\left(a_{s}-a_{f}\right)} \\
1 & \hat{u}_{1}=\frac{b_{s}+a_{s}-2 \rho}{2\left(b_{f}-a_{f}\right.}, \hat{u}_{2}=0 & \text { for } \frac{a_{s} b_{f}-a_{f} b_{s}}{\left(b_{f}-b_{s}\right)+\left(a_{s}-a_{f}\right)} \leq \rho<b_{f} \\
{[0,1]} & \hat{u}_{1}=\hat{u}_{2}=0 & b_{f} \leq \rho
\end{array}\right.
$$

where, as for class 1 , in the last range, $\rho \geq b_{f}$, the probability that the portfolio's marginal efficiency is below the threshold is 1 . 
Class 3: $a_{f}<a_{s}<b_{f}<b_{s}$

As for class 2, it turns out the degree of overlap of the two regions effects the solutions to the problem. We therefore again make some additional assumptions, $a_{s}<\left(b_{f}+a_{f}\right) / 2<\left(b_{s}+a_{s}\right) / 2<$ $b_{f}$, to fix the overlap to a particular region. The optimal allocation is then,

$$
\hat{x}=\left\{\begin{array}{lll}
{\left[0, \min \left\{1, \frac{a_{s}-\rho}{a_{s}-a_{f}}\right\}\right]} & \hat{u}_{1}=\hat{u}_{2}=0 & \text { for } \rho<a_{s} \\
\frac{\rho-a_{s}}{2 \rho-a_{f}-a_{s}} & \hat{u}_{1}=\hat{u}_{2}=0 & \text { for } a_{s} \leq \rho \leq \frac{1}{2}\left(b_{f}+a_{f}\right) \\
0 & \hat{u}_{1}=0, \hat{u}_{2}=\frac{2 \rho-b_{f}-a_{f}}{2\left(b_{s}-a_{s}\right)} & \text { for } \frac{1}{2}\left(b_{f}+a_{f}\right) \leq \rho<b_{s} \\
{[0,1]} & \hat{u}_{1}=\hat{u}_{2}=0 & b_{s} \leq \rho
\end{array}\right.
$$

where, again, as per the previous 2 classes, in the last range, $\rho \geq b_{f}$, the probability of actually satisfying the minimum threshold is 0 .

\section{Appendix S3: Derivation of marginal cost expressions}

\section{Management of koala population case study}

This work was published in Baxter et al. (2006) and is presented here again only for completeness. Let $N_{i}$ be the number of individuals in stage $i=2, \ldots, 9$, and $N$ be the total number of individuals in the adult population, stages $2-9$. Also let $B$, be the total available budget. We assume that both management actions target any individual in stages $2-9$ with equal probability. Then, for fecundity management, if darting one individual costs $c_{f}$ dollars, investing the entire budget in fecundity management will result in allocation of $B_{i}=B N_{i} / N$ dollars to stage $i$, treating $B_{i} / c_{f}$ individuals. Defining the per individual of stage $i$ fecundity as $f_{i}:=w_{i} f$, the number of young born to stage $i$ after fecundity management is therefore $N_{i} \tilde{f}_{i}=w_{i} f\left(N_{i}-B_{i} / c_{f}\right)$. 
Then, the reduction in fecundity rate of individuals in stage $i$ is given by

$$
\Delta f_{i}=\tilde{f}_{i}-f_{i}=w_{i} f B_{i} /\left(c_{f} N_{i}\right)
$$

The marginal cost of contraceptives in stage $i$ is thus $\partial C_{f_{i}} / \partial f_{i} \approx B_{i} / \Delta f_{i}=c_{f} N_{i} / w_{i} f$, and the marginal cost of contraceptives in all classes is

$$
\frac{\partial C_{f}}{\partial f}=\sum_{i=2}^{9} \frac{\partial C_{f}}{\partial C_{f_{i}}} \frac{\partial C_{f_{i}}}{\partial f_{i}} \frac{\partial f_{i}}{\partial f}=\sum_{i=2}^{9} \frac{c_{f} N_{i}}{w_{i} f} p_{i}=\frac{c_{f} N}{f}
$$

where we used the fact that $C_{f}=\sum_{i} C_{f_{i}}$.

Following similar logic for translocation management, if removing one individual costs $c_{s}$ dollars, a translocation budget of $B$ dollars acting pro rata among stages will remove $B_{i} / c_{s}$ individuals in stage $i$. The respective changes in number of individuals of stage $i$ advancing to next stage, and those remaining in the same stage are $\gamma_{i} q_{i} s\left(N_{i}-B_{i} / c_{s}\right)$, and $\left(1-\gamma_{i}\right) q_{i} s\left(N_{i}-\right.$ $\left.B_{i} / c_{s}\right)$. Thus the reduction in the per individual survival rate in stage $i, s_{i}=q_{i} s$, is

$$
\Delta s_{i}=\tilde{s}_{i}-s_{i}=\gamma_{i} q_{i} s B_{i} / c_{s} N_{i}+\left(1-\gamma_{i}\right) q_{i} s B_{i} / c_{s} N_{i}=q_{i} s B_{i} / c_{s} N_{i}
$$

The marginal cost of translocation in stage $i$ is therefore $\partial C_{s_{i}} / \partial s_{i} \approx B_{i} / \Delta s_{i}=c_{s} N_{i} / q_{i} s$, and the marginal cost of translocation in all stages is

$$
\frac{\partial C_{s}}{\partial s}=\sum_{i=2}^{9} \frac{\partial C_{s}}{\partial C_{s_{i}}} \frac{\partial C_{s_{i}}}{\partial s_{i}} \frac{\partial s_{i}}{\partial s}=\sum_{i=2}^{9} \frac{c_{s} N_{i}}{q_{i} s} q_{i}=\frac{c_{s} N_{2-9}}{s} .
$$

eqns. (S3.2)-(S3.4) are then used in eqns. (6) and (7) to obtain an expression for the marginal efficiency of each management action. 


\section{Conservation of sea turtle population case study}

We obtained the following conservation costs (in USD a year) and population data for an olive ridley sea turtle population in Orissa, India (personal communication). Nesting habitat protection cost was estimated to be between $\$ 1000$ and $\$ 10000$. For these costs the estimated increase in survival of hatchlings was $10 \%-50 \%$. Nesting habitat protection included protection of nests and eggs, protection of hatchlings, and elimination of disturbing artificial lighting.

Inclusion of TEDs (turtle exclusion devices) in the relevant fisheries was estimated to cost between $\$ 100000$ and $\$ 1000000$. For these costs the estimated increase in survival of nesting adults was estimated $\% 10-\% 25$. We also obtained nesting population size, and yearly number of adults stranded in fishing nets, estimates. Using these two estimates we confirmed the increase in adult survival estimate of $\% 10-\% 25$.

Next, we assumed that the marginal cost of either action is constant, and we let $h_{0}$ and $s_{0}$ denote the initial underlying survival parameter that is effected by conservation action for hatchlings and adults respectively. The minimum and maximum marginal cost estimates were therefore

$$
\begin{array}{ll}
\min \frac{\partial C_{h}}{\partial h}=\frac{1000}{0.5 h_{o}} & \max \frac{\partial C_{h}}{\partial h}=\frac{10000}{0.1 h_{o}} \\
\min \frac{\partial C_{s}}{\partial s}=\frac{10^{5}}{0.25 s_{o}} & \max \frac{\partial C_{s}}{\partial s}=\frac{10^{6}}{0.1 s_{o}}
\end{array}
$$

The marginal costs were then combined with the sensitivities to change in the respective conservation action obtained from Eq. (3), together with values for $h_{0}$ and $s_{0}$ from the population matrix (Table 2), to produce expressions for the stochastic marginal efficiency for each conservation action. 


\section{Appendix S4: Robustness to the linearity assumption in the portfolio model}

In constructing the portfolio, Eq. (1), we implicitly made the assumption that the marginal efficiency of the portfolio was a linear combination of the marginal efficiencies of each strategy. Furthermore, by only considering the fraction of the budget allocated to each strategy, and not the actual amount allocated, we made a second implicit assumption that the marginal efficiency scales linearly with respect to the total amount allocated. Both of these assumptions are likely to be incorrect in general. The dominant eigenvalue is not in general a linear function of the underlying parameters. In fact, it is not even an everywhere analytic function of such parameters. For example, in the case of the management of the koala population on Snake Island, the dominant eigenvalue is given by the largest magnitude root of the characteristic equation

$$
\phi(\lambda)=f\left(\sum_{j=1}^{8}(-1)^{j} w_{j+1} \prod_{i=1}^{j} \gamma_{i} q_{i} s \prod_{k=j+2}^{9}\left(\left(1-\gamma_{k}\right) q_{k} s-\lambda\right)\right)-\lambda \prod_{i=2}^{9}\left(\left(1-\gamma_{i}\right) q_{i} s-\lambda\right)=0
$$

Equation (S4.1) is a polynomial of degree 9 in $\lambda$. From Perron-Frobenius theory we know that the dominant eigenvalue is real and positive, but we cannot obtain an explicit expression for it. Instead we compute it numerically for each given combination of values for $f$ and $s$.

We therefore performed a sensitivity analysis to test robustness of our reported results. We now demonstrate such analysis for the case of management of koala population on Snake Island. In order to test the linearity assumptions we first express $f$ and $s$ in terms of the budget allocated to each strategy. Let $B_{f}=x B$ and $B_{s}=(1-x) B$ be the amount of the total budget $B$ allocated to the contraception and relocation strategy respectively. Then, assuming the budget is allocated 
pro rata across stages, the amount allocated to each stage for each strategy is given by

$$
B_{f, i}=x B N_{i} / N \quad \text { and } \quad B_{s, i}=(1-x) B N_{i} / N
$$

Adding " " " to any parameter to denote its value after management action, and using eqns. (S3.1) and (S3.3) and the definitions for $f_{i}$ and $s_{i}$, we have

$$
\begin{aligned}
& \tilde{f}=\frac{\tilde{f}_{i}}{w_{i}}=f\left(1-\frac{x B}{c_{f} N}\right), \\
& \tilde{s}=\frac{\tilde{s}_{i}}{q_{i}}=s\left(1-\frac{(1-x) B}{c_{s} N}\right) .
\end{aligned}
$$

We now vary $x \in[0,1]$ for a given budget $B$, and substitute the resulting values for $\tilde{f}$ and $\tilde{s}$ from eqns. (S4.3) and (S4.4) in the population matrix A. For each such matrix we then calculate numerically the dominant eigenvalue $\tilde{\lambda}$. Finally we compare the values for the expected marginal efficiency, $E[R(x)]$ (identical under both normal and uniform distributional assumption), with the observed marginal efficiency given by

$$
R_{o b s}(x):=\frac{\Delta \lambda}{B}=\frac{\tilde{\lambda}-\lambda}{B}
$$

where $\lambda$ is just the original dominant eigenvalue for the population prior to any management action.

Results for a set of four budget values $B \in\{0.00001,100,1000,10000\}$, and a fixed population size, $N=100$, are plotted in Fig. S4.1. First note that the numerical results are indeed approximately linear for budget values sufficiently low. In particular, from eqns. (S4.3) and (S4.4), the change in $f$ and $s$ are a function of $B / N$. From the numerical analysis, the linearity assumptions hold when $B$ and $N$ are of the same order of magnitude. For values of $B$ an order of magnitude larger than $N$, the numerical analysis is no longer linear however the 

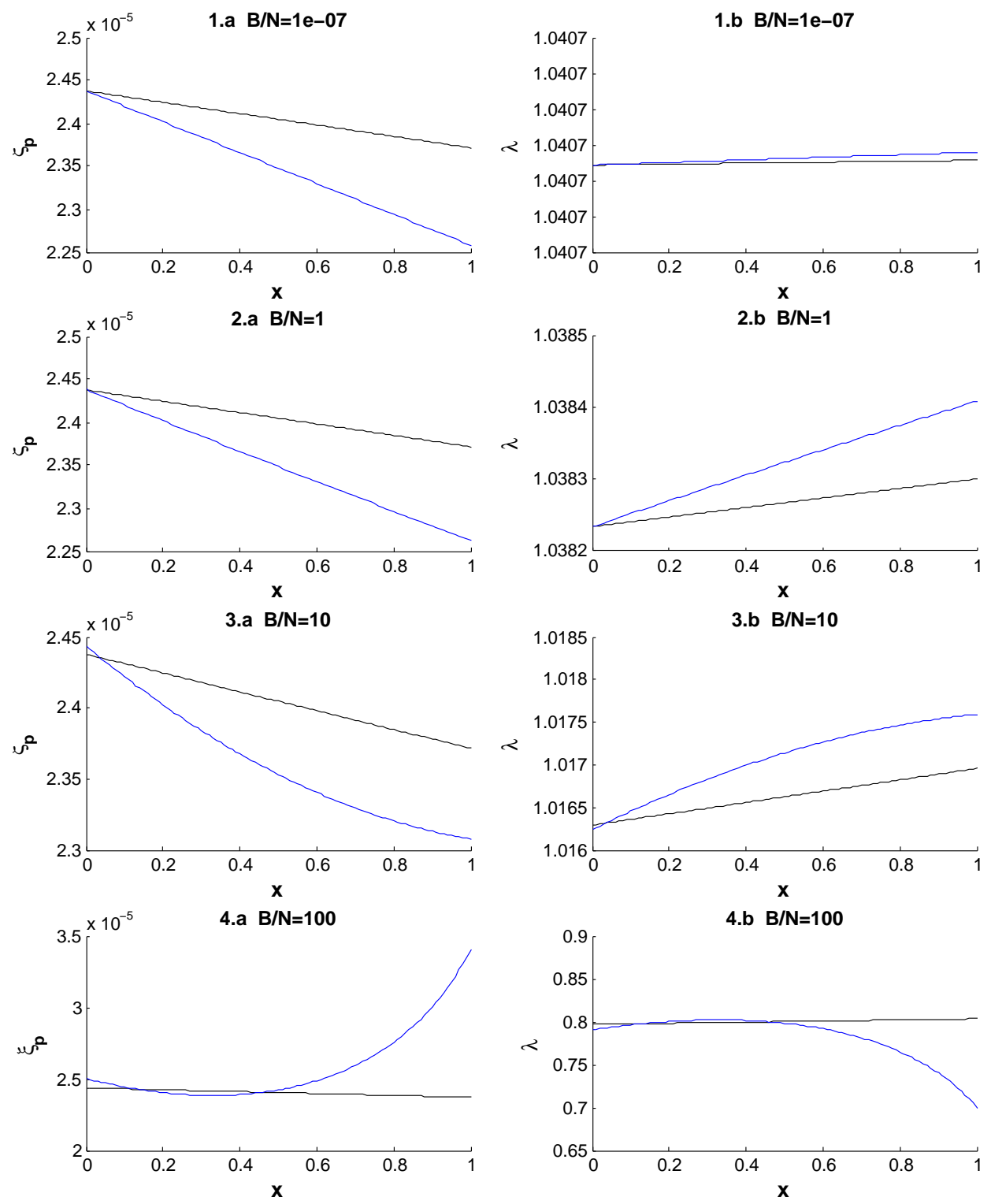

Figure S4.1: Plot of marginal efficiencies, 1a-4a, and dominant eigenvalue, $1 \mathrm{~b}-4 \mathrm{~b}$, for four total budget values. Black lines are predictions from model using linear assumptions and blue lines are results from direct numerical calculations $(\mathrm{N}=100)$. 
linear approximation is still an average approximation to the overall trend. That is, the linear approximation follows the general trend in the non linear behaviour. Beyond that, for values of $B$ more than an order of magnitude larger than $N$ the marginal efficiencies predicted by the linear model are a poor approximation of the actual dynamics of the system. Remembering that the per individual management costs are of the order of 100's of dollars, this budget translates to treating up to $10 \%$ of the population. 